\title{
Thermodynamic cost, speed, fluctuations, and error reduction of biological copy machines
}

\author{
Yonghyun Song ${ }^{1}$ and Changbong Hyeon ${ }^{1}$ \\ ${ }^{1}$ Korea Institute for Advanced Study, Seoul 02455, Korea
}

\begin{abstract}
Due to large fluctuations in cellular environments, transfer of information in biological processes without regulation is inherently error-prone. The mechanistic details of error-reducing mechanisms in biological copying processes have been a subject of active research; however, how error reduction of a process is balanced with its thermodynamic cost and dynamical properties remain largely unexplored. Here, we study the error reducing strategies in light of the recently discovered thermodynamic uncertainty relation (TUR) that sets a physical bound to the cost-precision trade-off relevant in general dissipative processes. We found that the two representative copying processes, DNA replication by the exonuclease-deficient T7 DNA polymerase and mRNA translation by the $E$. coli ribosome, reduce the error rates to biologically acceptable levels while also optimizing the processes close to the physical limit dictated by TUR.
\end{abstract}

Biological copying processes, which include DNA replication, transcription, and translation, have evolved errorreducing mechanisms to faithfully transmit information in the genetic code. In their seminal papers in the 1970s, Hopfield and Ninio [1, 2] proposed the kinetic proofreading mechanism to show that the energy-burning action of the mechanism can reduce the error rate. Shortly after, Bennett showed that the difference between kinetic barriers involving the incorporation of correct and incorrect substrates could be capitalized on to reduce the error rate under nonequilibrium chemical driving forces [3]. Despite differences in their mechanistic details, both models share a common feature that the reduction of copying error incurs free energy cost. Since these pioneering works, there have been a number of studies devoted to understanding the relation between the error reduction, speed, and energy consumption not only in the biological copying processes [4-9], but also in more general biochemical networks, including those related to sensory adaptation, circadian rhythm, and metabolic control [10-15].

Besides the faithful transmission of genetic information, the primary goal of biological copying processes is to generate biomass in the forms of DNA, RNA, and proteins. Intuitively, however, error reduction comes at the cost of energy dissipation or slowing down of the process. Furthermore, fluctuations in biomass synthesis, which concomitantly increase with heat dissipation for Michaelis-Menten type processes [16], also have to be suppressed below a biologically acceptable level. For instance, DNA replication in early fly embryogenesis occurs at high speed with exquisite precision; a modest change of $10 \%$ in replication timing could be lethal [17]. Similarly, for translation, it is well known that cells must express genes at the right protein copy number for optimal function in a given environment [18-20]; regulatory mechanisms are developed to suppress the copy number fluctuation in gene expression [21]. How biological processes balance these conflicting requirements is a fundamental subject to explore. To address such an issue, the recently developed thermodynamic uncertainty relation (TUR) [22], which offers a quantitative bound for dissipative processes at nonequilibrium steady states (NESS), is well suited.

TUR expresses the trade-off between the thermodynamic cost and uncertainty of dynamical processes in NESS and specifies its physical bound as follows:

$$
Q=q(t) \epsilon_{X}^{2}(t) \geq 2 k_{B} T
$$

This form of TUR holds for most of biological processes that can be represented either by stochastic jump processes on a kinetic network or by overdamped Langevin dynamics [23-26], though extensions to more general conditions, which adjust the lower bound of the original relation, have also been discussed in recent years [14, 27--34]. Briefly, $\epsilon_{X}(t) \equiv \sqrt{\left\langle\delta X(t)^{2}\right\rangle} /\langle X(t)\rangle$ is a relative uncertainty (or error) in an output observable $X(t)$ best representing the dynamic process at time $t$, and $q(t)$ denotes the thermodynamic cost or heat dissipation in generating the dynamic trajectory. The inequality in $\mathrm{Eq} 1$ allows one to quantitatively assess the physical limit to the precision that a dynamical process can maximize for a given amount of dissipation. Recently, $2 k_{B} T / Q$, which is bounded between 0 and 1 , was used to quantify the "transport efficiency" of molecular motors [25]. When $Q$ is written in the form of $Q=\dot{q}(t)\left(2 D / V^{2}\right)$ with $D$ and $V$ being the diffusivity and velocity of a molecular motor, the motor characterized with a small $Q$ can be interpreted as an efficient cargo transporter, because it transports cargos with high velocity $(V \sim\langle X(t)\rangle / t)$, small fluctuation $\left(D \sim\left\langle\delta X(t)^{2}\right\rangle / 2 t\right)$, but with small dissipation rate $(\dot{q})[35]$. Biosynthetic reactions that are efficient in suppressing fluctuations in product formation can also be characterized by small $Q$.

This work is organized into four parts. (i) We first introduce the basics of biological copying processes by reviewing the two distinct error reducing strategies by Bennett [3] and Hopfield [1]. (ii) We evaluate the error rate and $Q$ of the replication process by the exonuclease-deficient T7 DNA polymerase, a model process reminiscent of the kinetic discrimination mechanism by Bennett. (iii) We analyze a model of mRNA translation where both Bennett's kinetic discrimination and Hopfield's kinetic proofreading are employed to lower the error rate, and calculate $Q$ for translating a codon into a polypeptide chain. (vi) Lastly, we consider a more realistic model of mRNA translation that explicitly accounts for 42 types of aa-tRNA, and show that kinetic proofreading can suppress the fluctuation in the rate of polypeptide production. 


\section{RESULTS}

Error reducing mechanisms by Bennett and Hopfield. We briefly describe the two representative error reducing mechanisms, one by Bennett and the other by Hopfield. In a nutshell, the essence of the two mechanisms lies in an energydissipating enzymatic reaction of copy machines comprised of multiple kinetic cycles that can discriminate correct substrates from incorrect ones. Illustrated in Fig $1 \mathrm{~A}$ is an exemplary biological copying process where information of DNA sequence is copied by the DNA polymerase.

When the average reaction currents along the kinetic path associated with correct and incorrect substrate incorporation to the copy strand are defined as $\left\langle J^{c}\right\rangle$ and $\left\langle J^{i}\right\rangle$, respectively, the error probability, which will be discussed throughout this paper, is given by the ratio of two reaction currents

$$
\eta=\frac{\left\langle J^{i}\right\rangle}{\left\langle J^{c}\right\rangle+\left\langle J^{i}\right\rangle}
$$

Error reducing strategies of biological copying processes are at work to minimize $\eta$ to a level acceptable for the survival of an organism.

The mechanism of Bennett model (Fig 1] [3] uses the chemical potential of substrates, whose concentrations are kept out of equilibrium $\left(|\Delta \mu| \gg\left|\Delta \mu_{e q}\right|\right)$, as the free energy drive. In the model, correct and incorrect substrates are kinetically discriminated with different kinetic barriers, but with no difference in binding stabilities of the two substrate types. At equilibrium, $\left\langle J^{c}\right\rangle=\left\langle J^{i}\right\rangle=0$, and the error rate $\left(f=\left\langle J^{i}\right\rangle /\left\langle J^{c}\right\rangle\right)$ is solely determined by the ratio of equilibrium binding probabilities to copying system $\left(f_{0}=1\right)$, so that $\eta_{\text {eq }}=f_{0} /\left(1+f_{0}\right)=1 / 2$. When the free energy drive is large $(|\Delta \mu| \rightarrow \infty)$, the error rate converges to $\eta_{0}=1 /\left(1+e^{\beta \delta}\right)$, which is solely determined by the difference between the kinetic barriers for substrate binding, $\beta \delta$. Thus, as long as $\delta>0$, the mechanism can reduce the value of $\eta$ from $\eta_{e q}$ to $\eta_{0}$ at the expense of the free energy drive. See SI Text for the generalization of Bennett model where the equilibrium error rate is given by $f_{0}=e^{-\beta \Delta \mu_{i}} / e^{-\beta \Delta \mu_{c}}$.

Meanwhile, the original Hopfield model [1] (see Fig[1B) assumes that the binding rates $(\mathbf{E}+\mathbf{C} \rightarrow \mathbf{E C}$ or $\mathbf{E}+\mathbf{I} \rightarrow \mathbf{E I}$ in $\mathrm{Fig}, \mathrm{B}$ ) for the correct and incorrect substrates are identical $\left(k_{\mathrm{on}}^{c}[c]=k_{\mathrm{on}}^{i}[i]\right)$. In discriminating correct substrates from incorrect ones, the mechanism takes advantage of the facilitated unbinding of incorrect substrate from the copying system twice along the reaction path $(\mathbf{E I} \rightarrow \mathbf{E}+\mathbf{I}$ and $\mathbf{E I}^{*} \rightarrow \mathbf{E}+\mathbf{I}$ in Fig $1 \mathrm{~B}$, bottom), assisted by the extra free energy from molecular fuel consumption (GTP or ATP hydrolysis), which renders the reaction paths $\mathbf{E C} \rightarrow \mathbf{E C}^{*}$ and $\mathbf{E I} \rightarrow$ EI $^{*}$ effectively irreversible. The substrates complementary to the template polymer sequence are more likely to be polymerized, whereas the preferential unbinding of incorrect substrates from the copying complex end up with expending the energy for proofreading, giving rise to the futile cycle. The mechanism of Hopfield model, called the kinetic proofreading mechanism, reduces the error rate from $f_{0}$ down to $f_{0}^{2}$ [1].
A

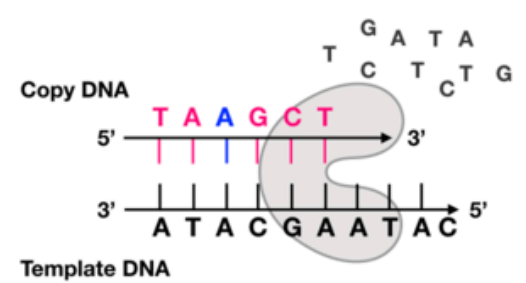

B
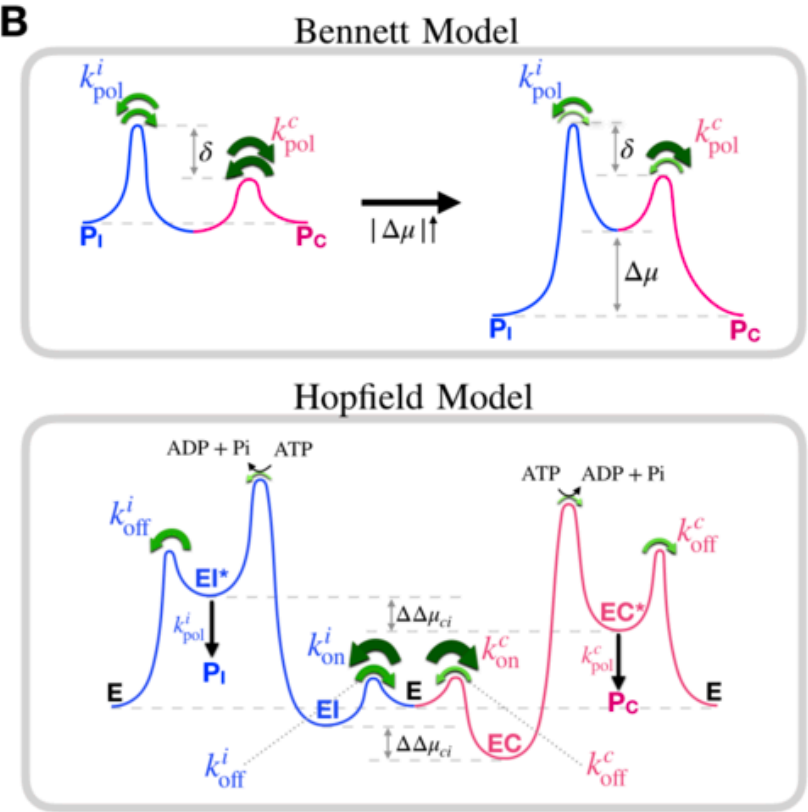

FIG. 1. The error reducing mechanisms of Bennett and Hopfield. (A) A cartoon illustrating a biological copying process using DNA replication. When the sequence of template DNA is copied, complementary (correct, $c$ ) or non-complementary (incorrect, $i$ ) nucleotide can be incorporated into the copy DNA. (B) (Top) The schematic of Bennett model [3]. Substrates are polymerized through a one-step enzyme reaction. Energetic input in the form of the chemical potential of the substrates $(\Delta \mu)$ leads to a larger current of correct substrate incorporation and reduces the error probability. (Bottom) The schematic of Hopfield model [1]. The substrates are polymerized through a three-state kinetic mechanism with intermediate states $\mathbf{E}$, $\mathbf{E C}$, and $\mathbf{E C}^{*}$ for correct substrate, or $\mathbf{E}, \mathbf{E I}$, and $\mathbf{E I}^{*}$ for incorrect substrate. The reactions $\mathbf{E C} \rightarrow \mathbf{E C}^{*}$ and $\mathbf{E I} \rightarrow \mathbf{E I}^{*}$, which expend the chemical energy of ATP hydrolysis, are effectively irreversible. This allows the copy process to select against the incorrect substrate through two chances of facilitated unbinding, thereby reducing the error probability. For both schematics, the thickness and color of the arrows represent the relative magnitude of the respective rate constants.

Real biological copying processes modify or combine the above two error-reducing strategies. More details on the different types of error reducing strategies and their combined effects can be found in refs. [36, 37].

Kinetic discrimination of dNTP by the T7 DNA polymerase. The DNA polymerase, in the absence of exonuclease activity, is an enzyme that adapts the kinetic discrimination mechanism to reduce errors in replication [5, 38, 39]. 
A
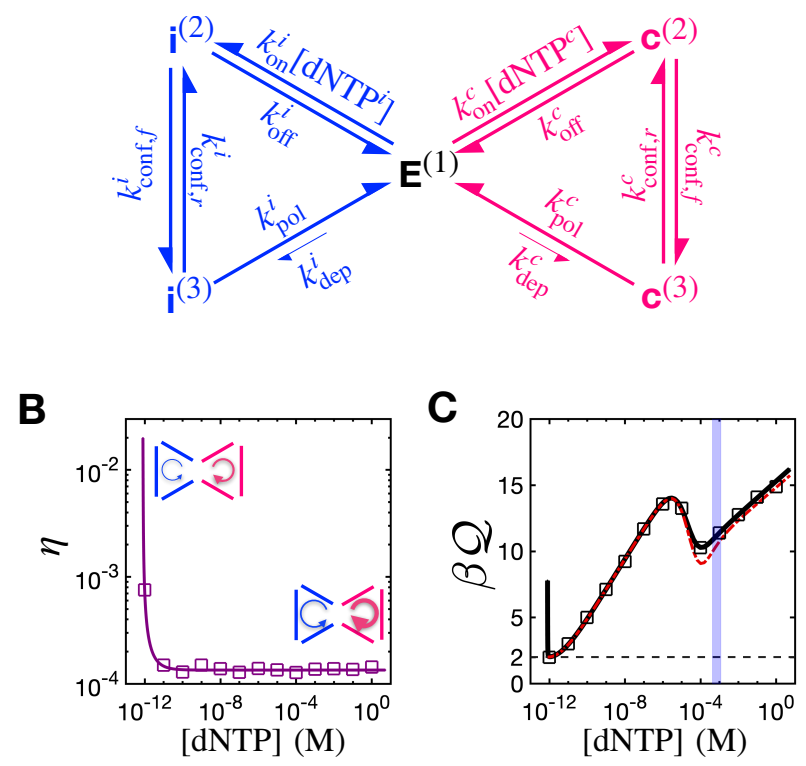

FIG. 2. The error reduction of DNA replication by the exonucleasedeficient T7 DNA polymerase. (A) The kinetic network for the dynamics of DNA polymerase [38]. $\left[\mathrm{dNTP}^{c}\right]$ and $\left[\mathrm{dNTP}^{i}\right]$ are the concentration of the correct and incorrect nucleotides, respectively, where $\left[\mathrm{dNTP}^{i}\right]=3\left[\mathrm{dNTP}^{c}\right]$ holds from the assumption that all 4 substrates are present at identical concentrations. (B) The error probability $(\eta)$ as a function of $[\mathrm{dNTP}]=\left[\mathrm{dNTP}^{c}\right]+\left[\mathrm{dNTP}^{i}\right]$. With increasing [dNTP], relatively more reaction current flows in the subcycle associated with correct nucleotide incorporation. (C) $Q$ of T7 DNA polymerase as functions of [dNTP]. The dash-dotted red line represents $Q$ of an analogously defined unicyclic network with rate constants corresponding to the correct nucleotide incorporation pathway. The range of dNTP concentrations in $E$. coli is demarcated with the purple shade [41-43]. For (B) and (C), the data points (squares) represent results from stochastic simulations using the Gillespie algorithm (see SI). See Fig. S5 for other related dynamical properties.

In its simplest form, the replication dynamics of DNA polymerases can be represented by a double-cyclic reversible 3state network consisting of two topologically identical subcycles for the incorporation of correct and incorrect nucleotides (Fig 2A). Following the binding of the substrate (dNTP) $([(1) \rightleftharpoons(2)])$, the polymerase on DNA undergoes conformational change $([(2) \rightleftharpoons(3)])$. Finally, the effectively irreversible polymerization associated with dNTP incorporation $([(3) \rightleftharpoons(1)])$ with $k_{\mathrm{pol}}^{c} \gg k_{\mathrm{dep}}^{c}$ and $k_{\mathrm{pol}}^{i} \gg k_{\mathrm{dep}}^{i}$, completes the kinetic cycle. The free energy difference between the binding of correct and incorrect nucleotides is approximately $\approx 5 k_{B} T$ [40], which implies that the error probability at equilibrium is $\eta_{e q} \approx 7 \times 10^{-3}$. In the presence of non-equilibrium drive, the conditions of $k_{\mathrm{conf}, f}^{c} \gg k_{\mathrm{conf}, f}^{i}$ and $k_{\mathrm{pol}}^{c} \gg k_{\mathrm{pol}}^{i}$, engendering much larger reaction current along $\mathbf{E}^{(1)} \rightleftharpoons \mathbf{c}^{(2)} \rightleftharpoons \mathbf{c}^{(3)} \rightleftharpoons \mathbf{E}^{(1)}$ than that along $\mathbf{E}^{(1)} \rightleftharpoons \mathbf{i}^{(2)} \rightleftharpoons \mathbf{i}^{(3)} \rightleftharpoons \mathbf{E}^{(1)}$, allows DNA polymerases to reduce $\eta$ below $\eta_{e q}[38,39]$.

As the total reaction current of polymerization, $\left\langle J_{\text {pol }}\right\rangle=$ $\left\langle J_{\text {pol }}^{c}\right\rangle+\left\langle J_{\text {pol }}^{i}\right\rangle$, is a natural output observable accessible, for instance, in single molecule experiments [44-46], we calculate $Q$ of DNA replication as (see Eq,1, and Materials and Methods)

$$
Q=\mathcal{A} \frac{\left\langle\delta J_{\mathrm{pol}}^{2}\right\rangle}{\left\langle J_{\mathrm{pol}}\right\rangle} .
$$

Alternatively, one could conceive choosing the current of correct sequence incorporation, $J^{c}$, as the output variable; however, unlike that of $J_{\mathrm{pol}}$, the measurement of $J^{\mathrm{c}}$ requires the explicit knowledge of the DNA sequence being synthesized, which is not readily accessible to an experimental observer. As long as $\eta$ is small, it is expected that $\left\langle J^{c}\right\rangle \approx\left\langle J_{\text {pol }}\right\rangle$, and $\left\langle\left(\delta J^{c}\right)^{2}\right\rangle \approx\left\langle\left(\delta J_{\text {pol }}\right)^{2}\right\rangle$; thus, choosing $J^{c}$ as the output variable instead of $J_{\text {pol }}$ will not significantly alter the value of $Q$.

The free energy cost for a single step of polymerization (affinity, $\mathcal{A}$ ) can be written as

$$
\begin{aligned}
\beta \mathcal{A} & =-\beta\left[(1-\eta) \Delta \mu_{c}+\eta \Delta \mu_{i}\right]-\eta \ln \eta-(1-\eta) \ln (1-\eta) \\
& \equiv-\beta \Delta \mu+I,
\end{aligned}
$$

where $\Delta \mu_{c}$ and $\Delta \mu_{i}$ are the chemical potential difference along the correct and incorrect and polymerization cycles, respectively. $\beta \mathcal{A}$ can be decomposed into the free energy gain $(-\beta \Delta \mu)$ and the Shannon-entropy (I) arising from the chance of incorporating correct versus incorrect monomers in the copy strand. It is noteworthy that although $I \leq I_{\max }(=\ln 2)$ is usually small compared to $-\beta \Delta \mu$, it represents a fundamental thermodynamic property associated with stochastic copying processes (see Eq. S21] [47].

We explore how $Q$ is affected when dNTP concentration ([dNTP]), which serves as a proxy for the chemical potential drive $(-\beta \Delta \mu$ in Eq. S10, increases. We assume that the four types of dNTPs (A, G, C, T) are maintained in solution at equal concentrations, and use experimentally determined kinetic rates of the exonuclease-deficient T7 DNA polymerase to calculate $\eta$ and $Q$ (see Table S1) [38]. With increasing [dNTP], the reaction current flows predominantly in one of the subcycles $\left(\left\langle J^{c}\right\rangle \gg\left\langle J^{i}\right\rangle\right)$, and $\eta$ decreases monotonically to values consistent with experimental measurements [5, 48] (Fig. 2B); by contrast, $Q$ displays non-monotonic variation (Fig. 2C). For $Q$, two minima are identified, one at $Q \approx 2$ $k_{B} T$, and the other at $Q \approx 10 k_{B} T$ ([dNTP] $\left.\approx 100 \mu \mathrm{M}\right)$, suggesting a complex interplay between the dissipation, current and its fluctuation. The suboptimal value of $Q$ with respect to substrate concentration was also observed in models of transport motors [35]. Notably, the latter minimum is found near the range of the in vivo [dNTP] in E. coli $(430-1200 \mu \mathrm{M}$ [41-43]) (Fig. 2C).

To understand the nature of the two minima of $Q$, we calculated $Q$ of an analogously defined unicyclic 3-state model with kinetic rates identical to those of the correct nucleotide incorporation cycle. The comparison between the $Q$ of the two models suggests: (i) the global minimum is formed near the DB condition [dNTP] $\approx \frac{k_{\text {of }}^{c} k_{\text {conf },}^{c} k_{\text {dep }}^{c}}{k_{\text {on }}^{c} k_{\text {con } f}^{c} k_{\text {pol }}^{c}}$; 
A

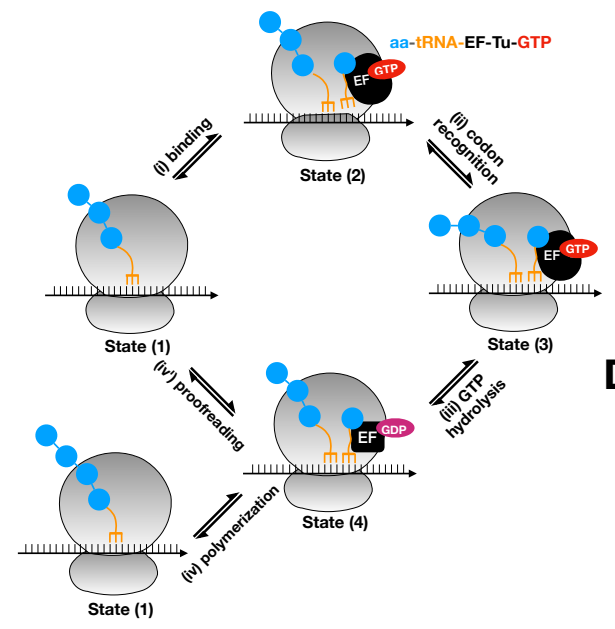

B
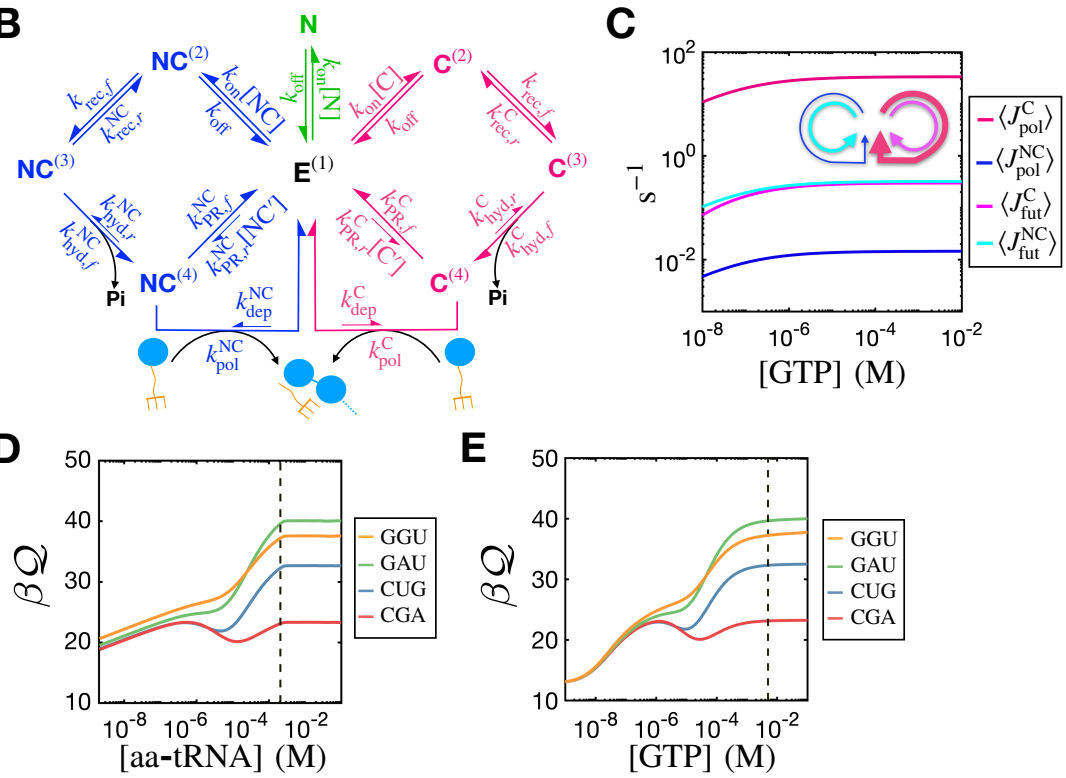

FIG. 3. mRNA translation. (A)(B) Schematics of the catalytic cycle of the E. coli ribosome. In (B), $\mathrm{C}^{(i)}$ and $\mathrm{NC}^{(i)}(i=2,3,4)$ represent intermediate states of the cognate and near-cognate aa-tRNA incorporation pathway. The state $\mathrm{N}$ represents the binding of the non-cognate aa-tRNA. $[\mathrm{C}],[\mathrm{NC}]$, and $[\mathrm{N}]$ represent the concentration of the cognate, near-cognate, and non-cognate ternary complex substrate, (aa-tRNA)(EF-Tu)-GTP, respectively. [ $\left.\mathrm{C}^{\prime}\right]$ and $\left[\mathrm{NC}^{\prime}\right]$ represent the concentration of the cognate and near-cognate (aa-tRNA)-(EF-Tu)-GDP, respectively. (C) The currents along the kinetic cycles as a function of [GTP] for codon CUG. The thickness of the lines in the inset schematic represents the relative magnitude of the reaction currents: $\left\langle J_{\text {pol }}^{\mathrm{C}}\right\rangle \gg\left\langle J_{\text {fut }}^{\mathrm{NC}}\right\rangle \gtrsim\left\langle J_{\text {fut }}^{\mathrm{C}}\right\rangle \gg\left\langle J_{\text {pol }}^{\mathrm{NC}}\right\rangle$. (D) $Q$ as a function of [aa-tRNA]. (E) $Q$ as a function of [GTP]. For (D) and (E), the dotted black line represents the cellular concentration in E. coli. See Fig. S6 for other related dynamical properties.

(ii) the other minimum at $[\mathrm{dNTP}] \approx 100 \mu \mathrm{M}$ arises from the Michaelis-Menten (MM) type enzyme kinetics. For Michaelis-Menten enzyme reactions, $Q$ is suboptimized when the substrate concentration is near the Michaelis-Menten constant $\left([S] \approx K_{m}\right)$, where the response of the reaction is maximal with respect to the logarithmic variation of substrate concentration (see SI text).

Simplified model of mRNA translation. Since its introduction by Hopfield and Ninio [1, 2], kinetic proofreading has been the most extensively discussed error reducing strategy [4, 8, 9, 37]. The proofreading reduces copy error by a resetting reaction that incurs an extra free energy. We study the effect of kinetic proofreading on $Q$ by taking mRNA translation of the E. coli ribosome as our model system (see Fig. 3).

The ribosome translates mRNA sequences into a polypeptide by reading codons, each consisting of three consecutive nucleic acids (Fig. 3A). When an aa-tRNA of a 'matching' codon binds to the ribosome-mRNA complex, the ribosome undergoes the reaction cycle for the cognate aa-tRNA incorporation (red cycle in Fig. 33 B). A near-cognate aa-tRNA with a single mismatch can also be incorporated, through a topologically identical but different kinetic pathway (blue cycle in Fig 3 B). For aa-tRNAs with two or three mismatches, corresponding to non-cognate aa-tRNAs, they can only interact with the ribosome-mRNA complex, but cannot undergo full incorporation (non-cognate aa-tRNA binding that corresponds to the reversible pathway colored in green in Fig. 3B B) [49, 50].

Translation by the ribosome occurs via the following steps: (i) an aa-tRNA is accommodated to the ribosome-mRNA complex in the form of the (aa-tRNA)-(EF-Tu)-GTP complex [(1) $\rightleftharpoons(2)]$, followed by (ii) the pairing of the codonanticodon sequence [(2) $\rightleftharpoons$ (3)]. (iii) GTP hydrolysis and the conformational change of EF-Tu $[(3) \rightleftharpoons$ (4)]. (iv) A new peptide bond formation with the ribosome translocating to the next codon $\left(k_{\mathrm{pol}}^{\mathrm{C}}\right.$ and $\left.k_{\mathrm{pol}}^{\mathrm{NC}}\right)$, or (iv') dissociation of (aatRNA)-(EF-Tu)-GDP complex from the ribosome (i.e. $k_{\mathrm{PR}, f}^{\mathrm{C}}$ and $k_{\mathrm{PR}, f}^{\mathrm{NC}}$ ). Both steps of (iv) and (iv') reset the system back to the state $(1)[(4) \rightleftharpoons(1)]$. The cognate aa-tRNAs are differentiated from near-cognate aa-tRNAs mainly due to the faster rates of GTP hydrolysis and peptide bond formation $\left(k_{\mathrm{hyd}, f}^{\mathrm{C}} \gg k_{\mathrm{hyd}, f}^{\mathrm{NC}}\right.$ and $\left.k_{\mathrm{pol}}^{\mathrm{C}} \gg k_{\mathrm{pol}}^{\mathrm{NC}}\right)$. The rates associated with tRNA binding, unbinding and recognition are similar between the two. As a result, the reaction current of incorporating the cognate aa-tRNA is greater than that of the near-cognate aa-tRNA along the network depicted in Fig. 3 B. Because the incorporation current of non-cognate aa-tRNA is effectively zero $\left(\left\langle J_{\mathrm{pol}}^{\mathrm{N}}\right\rangle=0\right)$, the error probability of the ribosome is $\eta=\left\langle J_{\text {pol }}^{\mathrm{NC}}\right\rangle /\left(\left\langle J_{\text {pol }}^{\mathrm{C}}\right\rangle+\left\langle J_{\text {pol }}^{\mathrm{NC}}\right\rangle\right)$, where $\left\langle J_{\text {pol }}^{\mathrm{C}}\right\rangle$ and $\left\langle J_{\text {pol }}^{\mathrm{NC}}\right\rangle$ are the currents of cognate and near-cognate aa-tRNA incorporations, respectively.

Similar to DNA replication, the free energy cost for a single 
step of translation $(\mathcal{A})$ can be written as

$$
\beta \mathcal{A}=-\beta\left[\Delta \mu_{\mathrm{pol}}+\frac{\left\langle J_{\mathrm{fut}}\right\rangle}{\left\langle J_{\mathrm{pol}}\right\rangle} \Delta \mu_{\mathrm{fut}}\right]-\eta \ln \eta-(1-\eta) \ln (1-\eta) .
$$

Here, $\Delta \mu_{\text {fut }}$ and $\Delta \mu_{\text {pol }}$ are the chemical potential difference along the futile and polymerization cycles, respectively (see SI for details). The kinetic proofreading uses extra energy in the form of GTP hydrolysis $\left(\Delta \mu_{\mathrm{fut}}\right)$, engendering futile cycles, and reduces $\eta$ further than that by kinetic discrimination alone, the latter of which only capitalizes on the thermodynamic cost of polymerization $\left(\Delta \mu_{\mathrm{pol}}\right)$.

The dynamics of mRNA translation was examined as a function of the concentration of aa-tRNA and GTP by assuming that the ternary complex concentration was in pseudoequilibrium with respect to the concentration of its components, aa-tRNA, EF-Tu, GTP, and GDP (see SI). With increasing [GTP], the polymerization current of all cycles increases while maintaining their relative magnitudes: $\left\langle J_{\text {pol }}^{\mathrm{C}}\right\rangle \gg\left\langle J_{\text {fut }}^{\mathrm{NC}}\right\rangle \gtrsim$ $\left\langle J_{\text {fut }}^{\mathrm{C}}\right\rangle \gg\left\langle J_{\text {pol }}^{\mathrm{NC}}\right\rangle$ (Fig. 3 3 ). In other words, while most cognate aa-tRNAs that reach state $\mathbf{C}^{(4)}$ are polymerized, most of the near-cognate aa-tRNAs that reach state $\mathbf{N C}^{(4)}$ are rejected by the proofreading reaction.

For all codon types, $\eta$ is nearly constant for a wide range of [aa-tRNA] and [GTP] (Figs. S6A, E). In contrast, the shape of $Q$ varies depending on the codon (Figs. 3P, E). For most codons, $Q$ increases monotonically with [aa-tRNA] and [GTP]. For codons CGA and CUG, $Q$ has a local minimum at [aa-tRNA] $\approx 10 \mu \mathrm{M}$ and [GTP] $\approx 10 \mu \mathrm{M}$. The distinguishing feature of the codons CGA and CUG is their high cognate to near-cognate aa-tRNA concentration ratios $([\mathrm{C}] /[\mathrm{NC}] \approx 0.9$ for CGA and $[\mathrm{C}] /[\mathrm{NC}] \approx 0.5$ for CUG. Fig. S7], which suggests that the local minimum of $Q$ occurs when the contribution from the near-cognate incorporation pathway is relatively low. As seen in the case of T7 DNA polymerase (Fig. 2C and Fig. S5, the local minimum of $Q$ (Figs $3 \mathrm{D}, \mathrm{E}$ ), if any, is identified at regions where the response of $\left\langle J_{\mathrm{pol}}\right\rangle$ is large with respect to the logarithmic variation of [aa-tRNA] or [GTP] (Figs. S6B, F).

Multicyclic model of mRNA translation. To address the mRNA translation in a more realistic fashion, we consider a multicyclic model which translates 42 species of aa-tRNAs into 20 different amino-acids (Fig. 4). For each codon, the 42 aa-tRNAs are grouped into cognate, near-cognate and noncognate types (Fig. S7). Using the information on the concentration of 42 aa-tRNAs and the model illustrated in Fig. 4A, we simulated the translation of the tufB mRNA sequence consisting of $n_{\mathrm{aa}}=394$ amino-acids, which encodes for EF-Tu, a highly abundant protein in E. coli [51] (Fig. 4).

The dynamics arising from the multicyclic model are studied using an ensemble of trajectories generated from Gillespie simulations (Fig. 4B B). The total number of translational steps $\left(N_{t l n}\right)$ that complete the polymerization of the full amino-acid sequences varies from one realization to another. Selecting the completion time of translation $(\mathcal{T})$ as the output observable for each dynamic process, we define TUR of translation as

$$
Q=\left[-\Delta \mu+\beta^{-1} I\right] \frac{\left\langle(\delta \mathcal{T})^{2}\right\rangle}{\langle\mathcal{T}\rangle^{2}},
$$

where, similar to all previous models, the dissipation has contributions from the free energy drive $(\Delta \mu)$ and Shannonentropy $(I)$. Denoting the forward and reverse rate constants of each kinetic step by $k_{i, f}$ and $k_{i, r}$ for $i=1, \ldots, N_{t l n}$, we can compute the average free energy drive by $-\beta \Delta \mu=$ $\left\langle\sum_{i=1}^{N_{t h n}} \ln \left(k_{i, f} / k_{i, r}\right)\right\rangle$, where $\langle\ldots\rangle$ denotes the average over the ensemble of $10^{4}$ realizations. The entropic contribution can be computed as $I=-\sum_{l=1}^{n_{\mathrm{aa}}} \sum_{i_{\mathrm{aa}}=1}^{20} \eta_{i_{\mathrm{aa}}}^{l} \ln \eta_{i_{\mathrm{aa}}}^{l}$, where $\eta_{i_{\mathrm{aa}}}^{l}$ is the probability of incorporating one of the 20 types of amino-acids, at the $l$-th position.

Using the multicyclic model, we evaluated $\eta$ and $Q$ with respect to perturbations to the proofreading reaction, by considering a multiplication factor $\kappa_{\mathrm{PR}}$ to the original wild-type (WT) rate constants $k_{\mathrm{PR}, f}^{\mathrm{C}}, k_{\mathrm{PR}, r}^{\mathrm{C}}, k_{\mathrm{PR}, f}^{\mathrm{NC}}$, and $k_{\mathrm{PR}, r}^{\mathrm{NC}}$. Although the rate constants are not experimentally tunable parameters like [GTP], the cell can optimize them throughout the evolution by means of mutations to the ribosome, EF-Tu, and tRNA. This type of perturbative analysis can be used to decipher which feature of the reaction kinetics for mRNA translation is optimized in the cell (see the effect of other perturbations in Fig. S8.

The WT level of proofreading gives rise to an average speed $\left\langle J_{\text {pol }}\right\rangle \approx 16$ aa/sec and error probability $\eta \approx 10^{-3}$ in our simulation, consistent with the experimental measurements [52, 53]. While $\eta$ decreases monotonically with $\kappa_{\mathrm{PR}}, Q$ is nonmonotonic with $\kappa_{\mathrm{PR}}$, minimized near the wild type condition. At $\kappa_{\mathrm{PR}}=1$ we obtain $Q \approx 45 k_{B} T$ [54] (Fig. 4D). For the given kinetic parameters from WT, $Q$ is minimized to $\sim 30$ $k_{B} T$ when the rates of proofreading is augmented by 5 fold. In a scenario of negligibly low proofreading $\left(\kappa_{\mathrm{PR}}=10^{-2}\right)$, the completion times for the translation display a much broader distribution than that by the WT $\left(\kappa_{\mathrm{PR}}=1\right)$. Thus, near the WT condition, proofreading can simultaneously improve the fidelity of translation and suppress the fluctuation of protein synthesis in an energetically efficient way.

Importantly, fluctuations in the completion time for mRNA translation can be critical, as it could in turn lead to significant variation in protein copy number. Thus, our results demonstrate that kinetic proofreading, an error reducing strategy, can also contribute to the energetically efficient control of protein levels.

\section{DISCUSSION}

Implications of the T7 DNA polymerase model. In the wild type T7 DNA polymerase, the proofreading activity of the exonuclease further reduces $\eta$ by two orders of magnitude [55]. In fact, in more complex systems such as DNA 

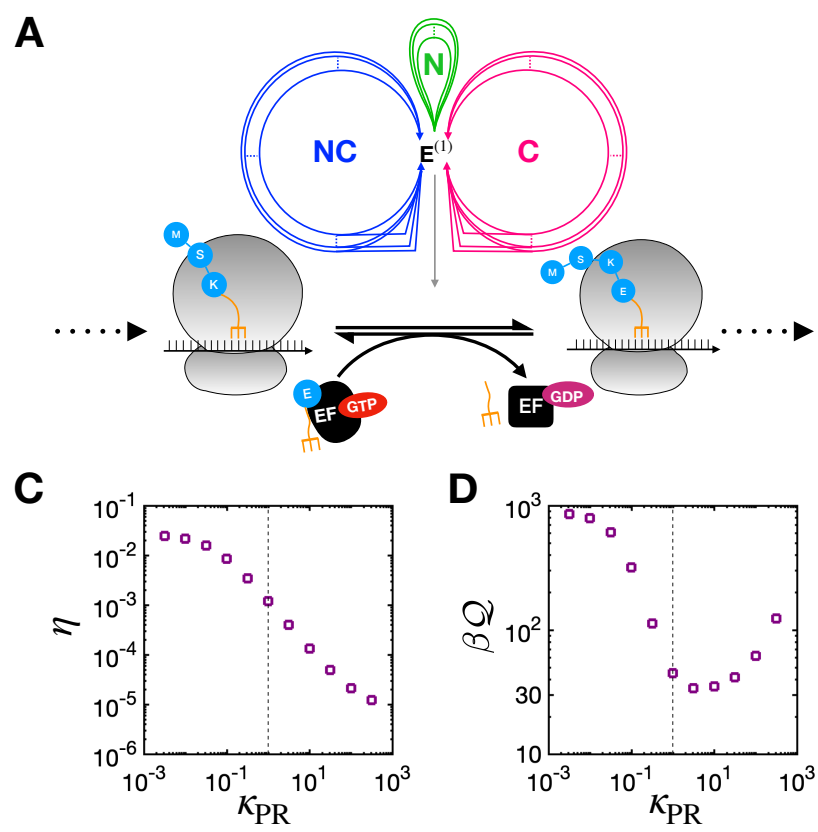

B
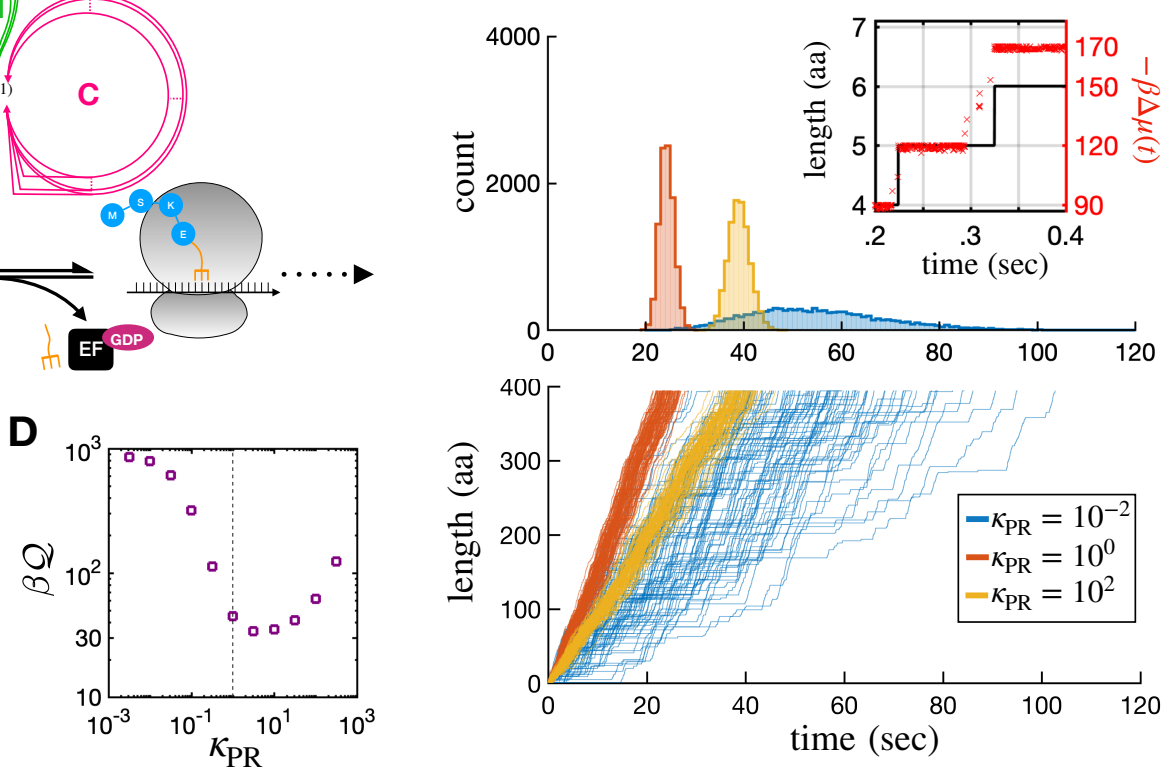

FIG. 4. The reaction kinetics of translation with 42 aa-tRNA species. (A) Schematic of the translation of the tufB mRNA sequence into EF-Tu. For each reaction cycle, 42 different aa-tRNAs can bind to the apo state of the enzyme. Out of these, the cognate and near cognate aa-tRNAs can undergo the reaction cycle previously defined in Figure 3 B. For more detail on the simulated reaction network, refer to the SI. (B) (Bottom) An ensemble of time traces $(N=100)$ generated from the numerics using Gillespie algorithm that simulates the mRNA translation (or the synthesis of the polypeptides consisting of 394 amino-acids) at different values of $\kappa_{\mathrm{PR}}$. (Top) The histogram of translation completion times. The inset shows a sample trajectory at the wild type condition $\left(\kappa_{\mathrm{PR}}=1\right)$, at which a proofreading reaction occurs at around 0.3 seconds. The average dissipation from the process is shown with red crosses $(\Delta \mu(t))$. Whenever the proofreading takes place, the synthesis of polypeptide is stalled. (C) The error probability and (D) $Q$ of TUR are plotted against $\kappa_{\mathrm{PR}}$. The dotted lines depict the wild type condition $\left(\kappa_{\mathrm{PR}}=1\right)$.

replication of E. coli, the combination of the actions of DNA polymerase, exonuclease, and mismatch repair machineries achieves an error probability as small as $\eta \approx 10^{-10}[56]$. Although these extra components of DNA replication could in principle be included in our model [3, 57,-59], general consensus on their kinetic network and measurement of kinetic rates are currently lacking. Thus, we focused on the simpler, yet still experimentally realizable, exonuclease-deficient $\mathrm{T} 7$ DNA polymerase, which has served as a useful tool for sequencing technologies and for biochemical studies of DNA polymerases [38, 60].

For the exonuclease-deficient T7 DNA polymerase, we found that $Q$ is suboptimized near the physiological [dNTP]. Similarly, it has recently been discovered that in metabolic reactions, the physiological substrate concentrations are generally close to their respective $K_{m}$ values [61]. A systems level analysis of yeast metabolism also showed that reaction currents of metabolism are generally self-regulated to the values at which their response to the change in substrate concentration is significant [62]. In light of our analysis of Michaelis-Menten enzyme reactions (see the section The suboptimal condition of reversible Michaelis-Menten reactions in SI), the above-mentioned condition of metabolism is closely related with the condition of suboptimized $Q$.
mRNA translation combines the strategies of kinetic discrimination and proofreading. The non-monotonic variation of $Q$ with $\kappa_{\mathrm{PR}}$ (Fig. 4D) is not a feature of the original kinetic proofreading model, which lacks the forward kinetic discrimination (i.e. $\beta \delta=0$ ). As the perturbative parameter $\kappa_{\mathrm{PR}}$ is increased, the error rate $\left(f=\left\langle J^{i}\right\rangle /\left\langle J^{c}\right\rangle\right)$ is reduced to $f \gtrsim f_{0}^{2}=e^{-2 \beta\left(\Delta \mu_{i}-\Delta \mu_{c}\right)}$ (Fig. S9A, blue line). Furthermore, in the original Hopfield model, $\lambda \approx 1$ regardless of $\kappa_{\mathrm{PR}}$ (Fig. S9P), which leads to $Q \approx \mathcal{A}$ (Fig. S9C, E), and a monotonically increasing $Q$ with $\kappa_{\mathrm{PR}}$ (Fig. $5 \mathrm{C}$, and Fig. $\mathrm{S} 9 \mathrm{E}$ ).

To introduce the kinetic discrimination to the Hopfield model, we consider a modified version, the associated kinetic constants of which satisfy the following relations with $\beta \delta>0$ :

$$
e^{\beta \delta}=\frac{k_{\mathrm{on}}^{c}}{k_{\mathrm{on}}^{i}}=\frac{k_{\mathrm{hyd}, f}^{c}}{k_{\mathrm{hyd}, f}^{i}}=\frac{k_{\mathrm{pol}}^{c}}{k_{\mathrm{pol}}^{i}}=\frac{k_{\mathrm{PR}, r}^{c}}{k_{\mathrm{PR}, r}^{i}} .
$$

As expected, $\eta$ decreases monotonically with $\beta \delta$ and $\kappa_{\mathrm{PR}}$ (Fig. 5B). Qualitatively similar to mRNA translation, $Q$ is minimized over a certain range of $\kappa_{\mathrm{PR}}$ as long as $e^{\beta \delta} \gtrsim 10^{1}$ (Fig. $5 \mathrm{~B}$ and Fig. $\mathrm{S9}$ E). Taken together with the modified Hopfield model, mRNA translation in E. coli balances the kinetic discrimination and proofreading, to attain low $\eta$ and suboptimized $Q$. 
A

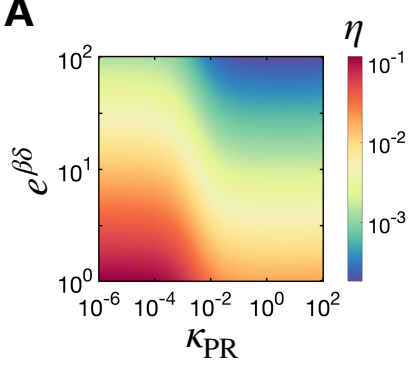

B

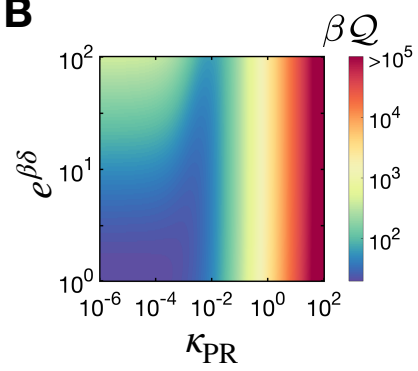

FIG. 5. The modified Hopfield model with kinetic discrimination. (A) The error probability ( $\eta$ ) and (B) $Q$ with respect to variations in $\delta$ and $\kappa_{\mathrm{PR}}$ defined in the main text. The rate constants used to generate the plots are given in Table S3. See Fig. S9 for other related dynamical properties plotted for $\beta \delta=0$ and $\beta \delta=\ln 10$.

Optimality of the speed and TUR in the E. coli ribosome. Similarly to our analysis shown in Fig. 4D, recent theoretical studies on mRNA translation by the ribosome [4, 6] have also observed that while the error probability is still far from its minimum, the WT value of the mean first translation time $\left(\left\langle\tau_{\text {MFPT }}\right\rangle\right)$ is close to its minimum; and hence it was concluded that the $E$. coli ribosome is primarily optimized for speed. As far as the $\kappa_{\mathrm{PR}}$-dependencies of speed $\left(\left\langle J_{\text {pol }}\right\rangle \approx\left\langle\tau_{\mathrm{MFPT}}\right\rangle^{-1}\right)$ and $\eta$ are concerned, our study points to the same finding (Fig. S8). In fact, recent studies, which showed translational pausing caused protein misfolding, lend support to the significance of optimal codon translation speed [63, 64].

Fast codon translation speed, small fluctuations in total translation time, and low thermodynamic costs could be favorable characteristics of translation, all likely under evolutionary selection pressure [65]; however, not all of these requirements can be fulfilled simultaneously. In this aspect, of great significance is our finding that the TUR measure of $E$. coli ribosome $\left(Q \approx 45 k_{B} T\right)$ for the wild type condition is in the vicinity of its minimum with respect to $\kappa_{\mathrm{PR}}\left(\sim 30 k_{B} T\right)$ (Fig. 4D).

Significance of small $Q$. The theoretical lower bound of TUR $\left(Q=2 k_{B} T\right)$ allows us to endow physical significance to the $Q$ values obtained for the two essential copy machines $\left(Q \approx 10 k_{B} T\right.$ for the T7 DNA polymerase and $Q \approx 45 k_{B} T$ for the $E$. coli ribosome). For instance, we can compare $Q$ of copying enzymes to molecular clocks, in which TUR is defined with respect to the tradeoff between the energetic cost and the uncertainty in the cycle duration. Marsland et al. have recently demonstrated that TUR of multiple types of biochemical oscillators severely underperform the $2 k_{B} T$ bound [14]. For the circadian KaiABC oscillator system, $\beta Q \approx O\left(10^{2}\right)$. This either implies that the precision of cycle periodicity is the key priority over the energy expenditure, or that this synthetic biochemical cycle is not optimally designed under the constraint of TUR. In contrast, biological motors that transport cargo along cytoskeletal filaments display small $\beta Q(\approx 7-15)$, simultaneously minimizing energetic costs, fluctuation, and

maximizing speed [35]. Compared to biological motors harnessing the thermal fluctuations along with the ATP hydrolysis free energy, synthetic nanomachines [66], which uses $\sim e V$ UV-light source as the driving force, are expected to have much greater $Q$ values. While the biological function of copying enzymes is to maintain low copying error, it is remarkable to discover that T7 DNA polymerase and E. coli ribosome are also working at conditions close to the theoretical bound dictated by the TUR.

\section{METHODS}

When the number of steps taken by the enzyme is selected as the output observable $(X(t)=n(t)$ in Eq11, TUR in Eq1 1 is modified to

$$
Q=q(t) \frac{\left\langle\delta n(t)^{2}\right\rangle}{\langle n(t)\rangle^{2}}=\mathcal{A} \lambda \geq 2 k_{B} T
$$

where $\mathcal{A}=q(t) /\langle n(t)\rangle$ and $\lambda=\left\langle\delta n(t)^{2}\right\rangle /\langle n(t)\rangle$ is the Fano factor of the copying process, which can also be written as $\lambda=\left\langle\delta J^{2}\right\rangle /\langle J\rangle$.

This work was supported by the KIAS Individual Grant No. CG067102 (Y.S.) and No. CG035003 (C.H.) at Korea Institute for Advanced Study. We thank the Center for Advanced Computation in KIAS for providing computing resources.

[1] J. Hopfield, Proc. Natl. Acad. Sci. 71, 4135 (1974).

[2] J. Ninio, Biochimie 57, 587 (1975)

[3] C. H. Bennett, BioSystems 11, 85 (1976).

[4] K. Banerjee, A. B. Kolomeisky, and O. A. Igoshin, Proc. Natl. Acad. Sci. 114, 5183 (2017)

[5] F. Cady and H. Qian, Phys. Biol. 6 (2009), 10.1088/14783975/6/3/036011

[6] J. D. Mallory, A. B. Kolomeisky, and O. A. Igoshin, J. Phys. Chem. B. 123, 4718 (2019).

[7] H. Mellenius and M. Ehrenberg, Nucleic Acids Research 45, 11582 (2017)

[8] A. Murugan, D. A. Huse, and S. Leibler, Proc. Natl. Acad. Sci. 109, 12034 (2012)

[9] R. Rao and L. Peliti, J. Stat. Mech.: Theory and Exp. 2015, P06001 (2015) arXiv:1504.02494

[10] Y. Cao, H. Wang, Q. Ouyang, and Y. Tu, Nat. Phys. 11, 772 (2015)

[11] D. Hartich, A. C. Barato, and U. Seifert, New J. Phys. 17 (2015), 10.1088/1367-2630/17/5/055026.

[12] P. François and G. Altan-Bonnet, J. Stat. Phys. 162, 1130 (2016)

[13] G. Lan and Y. Tu, J. Roy. Soc. Interface 10 (2013), 10.1098/rsif.2013.0489

[14] R. Marsland, W. Cui, and J. M. Horowitz, J. Roy. Soc. Interface 16 (2019), 10.1098/rsif.2019.0098.

[15] H. Qian and D. A. Beard, IEE Proceedings: Systems Biology 153, 192 (2006), arXiv:0511005v2 [arXiv:q-bio]

[16] W. Hwang and C. Hyeon, J. Phys. Chem. Lett. 8, 250 (2017). 
[17] N. J. Djabrayan, C. M. Smits, M. Krajnc, S. Tomer, S. Yamada, W. C. Lemon, P. J. Keller, C. A. Rushlow, and S. Y. Shvartsman, Curr. Biol. , 1 (2019)

[18] E. Dekel and U. Alon, Nature 436, 588 (2005)

[19] M. Scott, S. Klumpp, E. M. Mateescu, and T. Hwa, Mol. Syst. Biol. 10, 747 (2014)

[20] G.-W. Li, D. Burkhardt, C. Gross, and J. S. Weissman, Cell 157, 624 (2014)

[21] H. B. Fraser, A. E. Hirsh, G. Giaever, J. Kumm, and M. B. Eisen, PLoS Biol. 2, 834 (2004)

[22] A. C. Barato and U. Seifert, Phys. Rev. Lett. 114, 158101 (2015).

[23] T. R. Gingrich, J. M. Horowitz, N. Perunov, and J. L. England, Phys. Rev. Lett. 116, 120601 (2016).

[24] C. Hyeon and W. Hwang, Phys. Rev. E. 96, 012156 (2017).

[25] A. Dechant and S.-i. Sasa, Phys. Rev. E 97, 062101 (2018).

[26] S. Pigolotti, I. Neri, É. Roldán, and F. Jülicher, Phys. Rev. Lett. 119, 140604 (2017).

[27] S. Lee, C. Hyeon, and J. Jo, Phys. Rev. E 98, 032119 (2018).

[28] J. M. Horowitz and T. R. Gingrich, Phys. Rev. E 96, 020103 (2017).

[29] K. Brandner, T. Hanazato, and K. Saito, Phys. Rev. Lett. 120, 090601 (2018).

[30] A. C. Barato, R. Chetrite, A. Faggionato, and D. Gabrielli, New J. Phys. 20, 103023 (2018).

[31] H.-M. Chun, L. P. Fischer, and U. Seifert, Phys. Rev. E 99, 042128 (2019).

[32] Y. Hasegawa and T. Van Vu, Phys. Rev. Lett. 123, 110602 (2019).

[33] A. M. Timpanaro, G. Guarnieri, J. Goold, and G. T. Landi, Phys. Rev. Lett. 123, 090604 (2019).

[34] J. M. Horowitz and T. R. Gingrich, Nat. Phys. , 1 (2019).

[35] W. Hwang and C. Hyeon, J. Phys. Chem. Lett. 9, 513 (2018).

[36] P. Sartori and S. Pigolotti, Phys. Rev. Lett. 110, 1 (2013)

[37] S. Pigolotti and P. Sartori, J. Stat. Phys. 162, 1167 (2016)

[38] Y. C. Tsai and K. A. Johnson, Biochemistry 45, 9675 (2006)

[39] K. A. Johnson, Biochmi. Biophys. Acta 1804, 1041 (2010)

[40] M. F. Goodman, Proc. Natl. Acad. Sci. U. S. A. 94, 10493 (1997)

[41] B. R. Bochner and B. N. Ames, J. Biol. Chem. 257, 9759 (1982).

[42] M. H. Buckstein, J. He, and H. Rubin, J. Bacteriol. 190, 718 (2008)

[43] R. M. Schaaper and C. K. Mathews, DNA Repair 12, 73 (2013)

[44] E. A. Abbondanzieri, W. J. Greenleaf, J. W. Shaevitz, R. Landick, and S. M. Block, Nature 438, 460 (2005).

[45] J.-D. Wen, L. Lancaster, C. Hodges, A.-C. Zeri, S. H. Yoshimura, H. F. Noller, C. Bustamante, and I. Tinoco, Nature 452, 598 (2008).

[46] C. M. Kaiser, D. H. Goldman, J. D. Chodera, I. Tinoco, and C. Bustamante, Science 334, 1723 (2011).

[47] P. Sartori and S. Pigolotti, Phys. Rev. X 5, 1 (2015)

[48] T. A. Kunkel, S. S. Patel, and K. A. Johnson, Proc. Natl. Acad. Sci. U. S. A. 91, 6830 (1994).

[49] H. Dong, L. Nilsson, and C. G. Kurland, J. Mol. Biol. 260, 649 (1996)

[50] I. Wohlgemuth, C. Pohl, J. Mittelstaet, A. L. Konevega, and M. V. Rodnina, Phil. Trans. Roy. Soc. B: Biol. Sci. 366, 2979 (2011)

[51] Y. Ishihama, T. Schmidt, J. Rappsilber, M. Mann, F. U. Harlt, M. J. Kerner, and D. Frishman, BMC Genomics 9, 1 (2008)

[52] F. Bouadloun, D. Donner, and C. Kurland, The EMBO Journal 2, 1351 (1983)

[53] R. Young and H. Bremer, Biochem. J. 160, 185 (1976)
[54] W. D. Piñeros and T. Tlusty, Phys. Rev. E 101, 022415 (2020) arXiv:1911.04673.

[55] M. J. Donlin, S. S. Patel, and K. A. Johnson, Biochemistry 30, 538 (1991)

[56] R. M. Schaaper, J. Biol. Chem. 268, 23762 (1993).

[57] K. Banerjee, A. B. Kolomeisky, and O. A. Igoshin, Proc. Natl. Acad. Sci. U. S. A. 114, 5183 (2017).

[58] P. Gaspard, Phys. Rev. E 93, 1 (2016), arXiv:arXiv:1604.02554v1

[59] T. P. Hoekstra, M. Depken, S. N. Lin, J. Cabanas-Danés, P. Gross, R. T. Dame, E. J. Peterman, and G. J. Wuite, Biophys. J. 112, 575 (2017)

[60] B. Zhu, Front. Microbiol. 5, 1 (2014)

[61] J. O. Park, S. A. Rubin, Y.-F. Xu, D. Amador-Noguez, J. Fan, T. Shlomi, and J. D. Rabinowitz, Nat. Chem. Biol. 12, 482 (2016)

[62] S. R. Hackett, V. R. Zanotelli, W. Xu, J. Goya, J. O. Park, D. H. Perlman, P. A. Gibney, D. Botstein, J. D. Storey, and J. D. Rabinowitz, Science 354 (2016), 10.1126/science.aaf2786

[63] D. D. Nedialkova and S. A. Leidel, Cell 161, 1606 (2015).

[64] F. Trovato and E. P. OBrien, Biophys. J. 112, 1807 (2017).

[65] E. Ilker and M. Hinczewski, Phys. Rev. Lett. 122, 238101 (2019).

[66] T. Kudernac, N. Ruangsupapichat, M. Parschau, B. Maciá, N. Katsonis, S. R. Harutyunyan, K.-H. Ernst, and B. L. Feringa, Nature 479, 208 (2011).

[67] P. Gaspard and D. Andrieux, J. Chem. Phys. 141 (2014), $10.1063 / 1.4890821$

[68] P. Gaspard, Phys. Rev. E 93 (2016), 10.1103/PhysRevE.93.042419

[69] P. Gaspard, arXiv:2001.04923 , 1 (2020) arXiv:2001.04923

[70] A. Dechant and S.-i. Sasa, J. Stat. Mech.: Theory and Exp. 2018, 063209 (2018).

[71] Z. Koza, J. Phys. A. 32, 7637 (1999).

[72] D. T. Gillespie, J. Phys. Chem. 81, 2340 (1977).

[73] S. Rudorf, M. Thommen, M. V. Rodnina, and R. Lipowsky, PLoS Comput. Biol. 10 (2014), 10.1371/journal.pcbi.1003909.

[74] J. M. Berg, J. L. Tymoczko, and L. Stryer, Biochemistry, 5th ed. (W. H. Freeman and Company, 2002).

[75] B. R. Martin, Biopolymers 45, 351 (1998).

[76] R. B. Loftfield, Prog. Nucleic Acid Res. Mol. Biol. 12, 87 (1972)

[77] M. E. Evans, W. C. Clark, G. Zheng, and T. Pan, Nucleic Acids Res. 45, e133 (2017)

[78] G. Romero, V. Chau, and R. L. Biltonen, J. Biol. Chem. 260, 6167 (1985).

[79] K. B. Gromadski, H. J. Wieden, and M. V. Rodnina, Biochemistry 41, 162 (2002)

[80] B. D. Bennett, E. H. Kimball, M. Gao, R. Osterhout, S. J. Van Dien, and J. D. Rabinowitz, Nat. Chem. Biol. 5, 593 (2009)

[81] C. A. Minetti, D. P. Remeta, H. Miller, C. A. Gelfand, G. E. Plum, A. P. Grollman, and K. J. Breslauer, Proc. Natl. Acad. Sci. U. S. A. 100, 14719 (2003). 


\section{SUPPORTING INFORMATION}

\section{Bennett model: kinetic discrimination without proofreading}

In the non-proofreading model of copy processes introduced by Bennett [3], a copying enzyme synthesizes the complementary polymer strand by incorporating the monomers

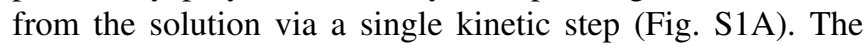
copy process is described by three key parameters: the binding free energies of correct and incorrect monomers, $\Delta \mu_{c}$ and $\Delta \mu_{i}$, and the difference in the kinetic barriers, $\delta$. Here, we keep the difference between the binding free energies $\left(\Delta \Delta \mu_{c i} \equiv \Delta \mu_{c}-\Delta \mu_{i}\right)$ and the difference between the kinetic barriers constant. For instance, in DNA replication, $\Delta \Delta \mu_{c i}$ and $\delta$ are determined by the molecular properties of the nucleotides and the polymerase. The error probability can be modulated by changing nucleotide concentrations, which corresponds to changing $\Delta \mu_{c}$ in the Bennett model. Thus, in the Bennett model, $\eta$ is evaluated as a function of $\Delta \mu_{c}$, and vice versa. In the following, we derive the expressions of $\Delta \mu_{c}(\eta)$, $\lambda(\eta), \mathcal{A}(\eta)$ and $Q(\eta)$. Using these expressions, we plot the diagrams of $\eta$ and $Q$ as functions of $\delta$ and $\Delta \mu_{c}$ (Fig. S1B-E).

The evolution of the probability $P$ of the complementary polymer can be described by the following master equation,

$$
\begin{aligned}
\dot{P}(\ldots c)= & k_{f}^{c} P(\ldots)+k_{r}^{c} P(\ldots c c)+k_{r}^{i} P(\ldots c i) \\
& -\left(k_{f}^{c}+k_{f}^{i}+k_{r}^{c}\right) P(\ldots c) \\
\dot{P}(\ldots i)= & k_{f}^{i} P(\ldots)+k_{r}^{c} P(\ldots i c)+k_{r}^{i} P(\ldots i i) \\
& -\left(k_{f}^{c}+k_{f}^{i}+k_{r}^{i}\right) P(\ldots i)
\end{aligned}
$$

where the sequence of the complementary polymer is represented by $(\ldots),(\ldots c),(\ldots i)$, and so on as in Fig. S1A.

Assuming that the error probability of each position of the copy polymer is independent from the prior sequence, we can make the following substitutions: $P(\ldots c)=P(\ldots) P(c)$, $P(\ldots c i)=P(\ldots) P(c) P(i)$, and so forth. Then, at steady state, Eq. S1 takes the form

$$
\begin{aligned}
k_{f}^{c}+k_{r}^{c} P(c)^{2}+k_{r}^{i} P(c) P(i)-\left(k_{f}^{c}+k_{f}^{i}+k_{r}^{c}\right) P(c) & =0 \\
k_{f}^{i}+k_{r}^{c} P(c) P(i)+k_{r}^{i} P(i)^{2}-\left(k_{f}^{c}+k_{f}^{i}+k_{r}^{i}\right) P(i) & =0 .
\end{aligned}
$$

By rearranging the above equations, we can write the error probability, $\eta$, as

$$
\eta=P(i)=\frac{\left\langle J^{i}\right\rangle}{\left\langle J^{i}\right\rangle+\left\langle J^{c}\right\rangle}=\frac{k_{f}^{i}-\eta k_{r}^{i}}{k_{f}^{i}-\eta k_{r}^{i}+k_{f}^{c}-(1-\eta) k_{r}^{c}},
$$

where $\left\langle J^{c}\right\rangle$ and $\left\langle J^{i}\right\rangle$ are defined as the average reaction currents for correct and incorrect monomers, respectively,

$$
\begin{aligned}
& \left\langle J^{c}\right\rangle=k_{f}^{c}-(1-\eta) k_{r}^{c} \\
& \left\langle J^{i}\right\rangle=k_{f}^{i}-\eta k_{r}^{i} .
\end{aligned}
$$

Essentially, we have transformed the dynamics along the tree structure (Fig S1A) into a Markov process. The equivalence
A
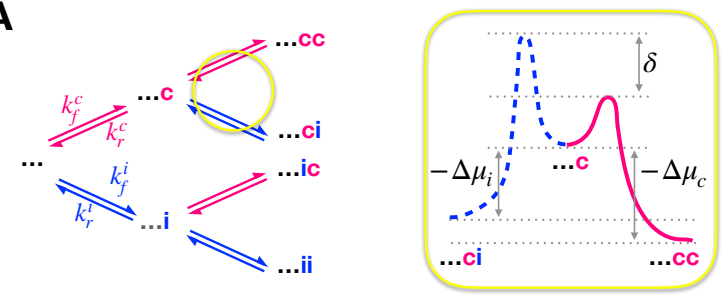

B

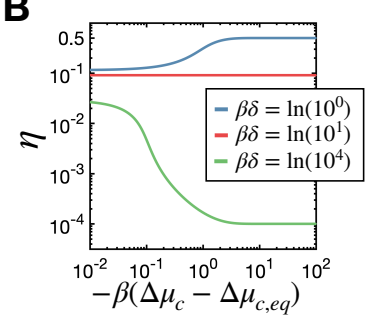

C
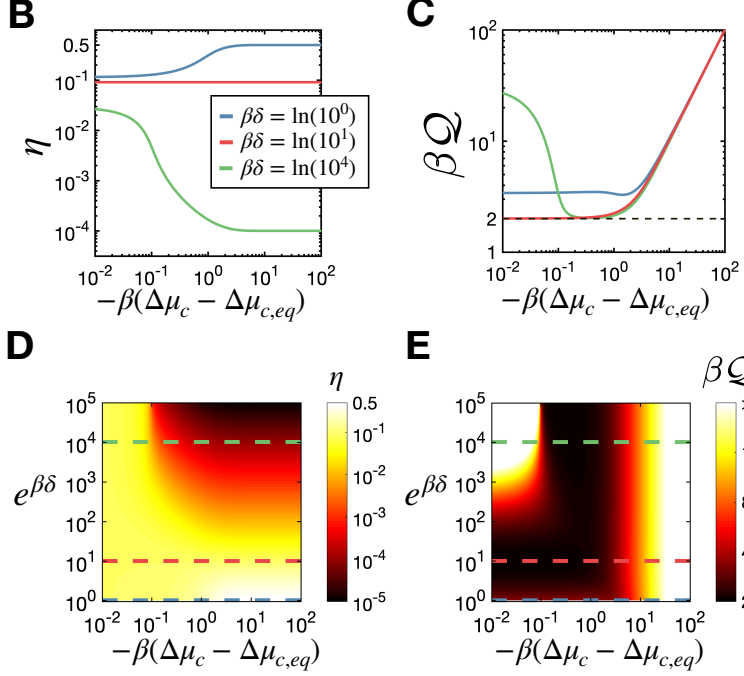

E

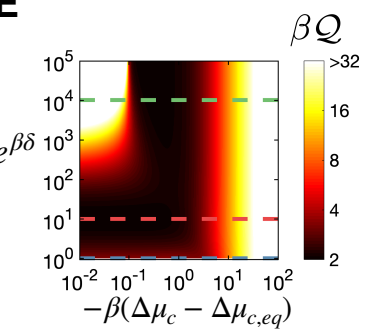

FIG. S1. The reaction kinetics of a non-proofreading copying system generalizing Bennett model. (A) (Left) The schematic of the copy polymer elongation. The (...) represents the previously synthesized copy polymer. (Right) The schematic of the free energy profile of monomer incorporation. The free energies of incorporating correct and incorrect monomers are $\beta \Delta \mu_{c}=-\ln \left(k_{f}^{c} / k_{r}^{c}\right)$ and $\beta \Delta \mu_{i}=-\ln \left(k_{f}^{i} / k_{r}^{i}\right)$, respectively. The incorporation of the correct monomer occurs faster than that of the incorrect monomer, as described by the parameter $\beta \delta=\ln \left(k_{f}^{c} / k_{f}^{i}\right)>0$. (B) The error probability $(\eta)$ and (C) $Q$ as functions of the chemical potential bias for different values of the kinetic discrimination parameter $(\beta \delta)$, where $e^{-\beta\left(\Delta \mu_{c}-\Delta \mu_{i}\right)}=10$. The curves in (C) for various $\beta \delta$ values are colorcoded identically as those in (B). In (C), the physical limit of $\beta Q=2$ is marked with a dashed line. Diagrams of (D) $\eta$ and (E) $\beta Q$ as a function of $e^{\beta \delta}$ and $-\beta\left(\Delta \mu_{c}-\Delta \mu_{c, e q}\right)$.

of the expressions of $\eta$ from Eq. S1 and Eq. S2 has been shown for the double-cyclic reversible 3-state nework model in Ref. [5]. More general treatment of the dynamics that occur along tree structures can be found in refs. [67-69]. In the present work, the main conclusions pertaining to $\eta$ (and $Q$ ) are further supported by explicit simulations of the master equation representation (Fig. 2 $\mathrm{C}$ and Fig. 4D).

At the detailed balance (DB) condition, no current should flow through both pathways associated with correct and incorrect monomer incorporations, i.e., $\left\langle J^{c}\right\rangle=0$ and $\left\langle J^{i}\right\rangle=0$; 
$\beta \mathcal{A}^{c}=\ln \left(\frac{k_{f}^{c}}{(1-\eta) k_{r}^{c}}\right)=0$ and $\beta \mathcal{A}^{i}=\ln \left(\frac{k_{f}^{i}}{\eta k_{r}^{i}}\right)=0$, which leads to

$$
1-\eta_{e q}=\left(\frac{k_{f}^{c}}{k_{r}^{c}}\right)_{e q} \equiv e^{-\beta \Delta \mu_{c, e q}}
$$

and

$$
\eta_{e q}=\left(\frac{k_{f}^{i}}{k_{r}^{i}}\right)_{e q} \equiv e^{-\beta \Delta \mu_{i, e q}}
$$

We note that in order for $\eta_{e q}$ to be in the range of $0<\eta_{e q}<1$, $\beta \Delta \mu_{c, e q}$ and $\beta \Delta \mu_{i, e q}$ should be positive, meaning that chemical potential bias of monomer incorporation is positive (uphill). Then, by taking the ratio between Eqs. $\mathrm{S} 4$ and $\mathrm{S} 5$, we obtain

$$
\eta_{e q}=\frac{1}{1+e^{-\beta \Delta \Delta \mu_{c i}}} \text {. }
$$

where $\Delta \Delta \mu_{c i}=\Delta \mu_{c, e q}-\Delta \mu_{i, e q}$. At the limit of strongly forward driven reactions, i.e., $k_{f}^{c} \gg(1-\eta) k_{r}^{c}$ and $k_{f}^{i} \gg \eta k_{r}^{i}$, Eq. $[\mathrm{S} 2]$ is led to

$$
\eta \rightarrow k_{f}^{i} /\left(k_{f}^{i}+k_{f}^{c}\right)=1 /\left(1+e^{\beta \delta}\right) \equiv \eta_{0} .
$$

Next, we can write Eq. S2 as

$$
\begin{aligned}
\eta & =\frac{k_{f}^{i} / k_{r}^{i}-\eta}{k_{f}^{i} / k_{r}^{i}-\eta+\left(k_{r}^{c} / k_{r}^{i}\right)\left(k_{f}^{c} / k_{r}^{c}-(1-\eta)\right)} \\
& =\frac{e^{-\beta \Delta \mu_{i}}-\eta}{\left(e^{-\beta \Delta \mu_{i}}-\eta\right)+e^{-\beta\left(\Delta \Delta \mu_{c i}-\delta\right)}\left(e^{-\beta \Delta \mu_{c}}-(1-\eta)\right)}
\end{aligned}
$$

where $k_{r}^{c} / k_{r}^{i}=e^{-\beta\left(\Delta \Delta \mu_{c i}-\delta\right)}$ was used. After some rearrangements, we can express $\Delta \mu_{c}$ as a function of $\eta, \eta_{0}$, and $\eta_{e q}$ as follows

$$
-\beta \Delta \mu_{c}=\ln \frac{\eta(1-\eta)\left(\eta_{e q}-\eta_{0}\right)}{\left(\eta-\eta_{0}\right) \eta_{e q}} .
$$

Next, $\mathcal{A}$ can be written as

$$
\begin{aligned}
\beta \mathcal{A}(\eta) & =\frac{1}{\left\langle J^{c}\right\rangle+\left\langle J^{i}\right\rangle}\left[\left\langle J^{c}\right\rangle \ln \frac{k_{f}^{c}}{(1-\eta) k_{r}^{c}}+\left\langle J^{i}\right\rangle \ln \frac{k_{f}^{i}}{\eta k_{r}^{i}}\right] \\
& =(1-\eta) \ln \frac{k_{f}^{c}}{(1-\eta) k_{r}^{c}}+\eta \ln \frac{k_{f}^{i}}{\eta k_{r}^{i}} \\
& =\underbrace{-\beta\left[(1-\eta) \Delta \mu_{c}+\eta \Delta \mu_{i}\right]}_{=-\beta \Delta \mu}+\underbrace{[-(1-\eta) \ln (1-\eta)-\eta \ln \eta]}_{=I} \\
& =\eta \ln \frac{\eta_{e q}(1-\eta)}{\eta\left(1-\eta_{e q}\right)}+\ln \frac{\eta\left(\eta_{e q}-\eta_{0}\right)}{\eta_{e q}\left(\eta-\eta_{0}\right)} .
\end{aligned}
$$

As expected, $\lim _{\eta \rightarrow \eta_{\text {eq }}} \beta \mathcal{A}(\eta)=0$ and $\lim _{\eta \rightarrow \eta_{0}} \beta \mathcal{A}(\eta)=\infty$, which means that $\eta$ approaches $\eta_{e q}$ and $\eta_{0}$ at the zero and infinite dissipation limits, respectively.

Importantly, $\mathcal{A}$ can be decomposed into two contributions (Eq. $\mathrm{S} 10):-\beta \Delta \mu$ is the free energy gain after the monomer incorporation, and $I$ is the Shannon information entropy arising from the chance of incorporating correct $(c)$ and incorrect (i) monomers to the copy strand. The information $(I)$ is maximized to $I=\ln 2$ when the odds of incorporating the correct and incorrect monomers is identical $(\eta=1 / 2)$, whereas $I=0$ if only the correct or incorrect monomers are incorporated. This implies that as long as the chemical potential of monomers in solution is constantly maintained, the process near the $\mathrm{DB}$ condition $(\beta \mathcal{A}=-\beta \Delta \mu+I \gtrsim 0)$ can still be driven by the entropy $I(\geq \beta \Delta \mu)$ even if the polymerization is energetically uphill $(\Delta \mu>0)$ [3].

The Fano factor $\lambda$ can be calculated as

$$
\begin{aligned}
\lambda(\eta) & =\frac{\left\langle\left(\delta J^{c}\right)^{2}\right\rangle+\left\langle\left(\delta J^{i}\right)^{2}\right\rangle}{\left\langle J^{c}\right\rangle+\left\langle J^{i}\right\rangle} \\
& =\frac{\left(k_{f}^{i}+\eta k_{r}^{i}\right)+\left(k_{f}^{c}+(1-\eta) k_{r}^{c}\right)}{\left(k_{f}^{i}-\eta k_{r}^{i}\right)+\left(k_{f}^{c}-(1-\eta) k_{r}^{c}\right)} \\
& =\frac{\left(k_{f}^{i} / k_{r}^{i}+\eta\right)+\left(k_{r}^{c} / k_{r}^{i}\right)\left(k_{f}^{c} / k_{r}^{c}+(1-\eta)\right)}{\left(k_{f}^{i} / k_{r}^{i}-\eta\right)+\left(k_{r}^{c} / k_{r}^{i}\right)\left(k_{f}^{c} / k_{r}^{c}-(1-\eta)\right)} \\
& =\frac{\left(e^{-\beta \Delta \mu_{i}}+\eta\right)+e^{-\beta\left(\Delta \Delta \mu_{c i}-\delta\right)}\left(e^{-\beta \Delta \mu_{c}}+(1-\eta)\right)}{\left(e^{-\beta \Delta \mu_{i}}-\eta\right)+e^{-\beta\left(\Delta \Delta \mu_{c i}-\delta\right)}\left(e^{-\beta \Delta \mu_{c}}-(1-\eta)\right)} \\
& =\frac{2\left(\eta_{e q}-\eta_{0}\right) \eta^{2}+\left(\eta_{0}+\eta_{0}^{2}-2 \eta_{e q}\right) \eta+\left(1-\eta_{0}\right) \eta_{0} \eta_{e q}}{\eta_{0}\left(1-\eta_{0}\right)\left(\eta-\eta_{e q}\right)} .
\end{aligned}
$$

$Q(\eta)$ evaluated using the expression of $\mathcal{A}(\eta)$ and $\lambda(\eta)$, i.e., $Q(\eta)=\mathcal{A}(\eta) \lambda(\eta)$, quantifies the translational efficiency of the copying enzyme along the template polymer [35, 70]. For strongly driven systems $(\mathcal{A} \gg 0$ ), all the curves of $Q(\mathcal{A})$ with different values of $\delta$ converge (Fig. S1C). However, near the DB condition, where $I$ contributes significantly to $\mathcal{A}, Q$ shows complex dependence on $\delta$.

At the DB condition,

$$
\beta Q\left(\eta_{e q}\right)=2+\frac{\left(\eta_{e q}-\eta_{0}\right)^{2}}{\left(1-\eta_{0}\right) \eta_{0}} \geq 2 .
$$

Thus, the lower bound $2 k_{B} T$ is attained at the DB condition when $\eta_{e q}=\eta_{0}$.

$Q$ can also approach its lower bound $2 k_{B} T$ at the limiting condition of $\beta \delta \gg 1$. At this limit, only correct monomers are incorporated into the copy polymer $\left(\left\langle J^{i}\right\rangle=0\right)$, which leads to

$$
\mathcal{Q}=\mathcal{A} \frac{\left\langle\left(\delta J^{c}\right)^{2}\right\rangle}{\left\langle J^{c}\right\rangle}=\mathcal{A} \frac{k_{f}^{c}+k_{r}^{c}}{k_{f}^{c}-k_{r}^{c}}=\mathcal{A} \frac{e^{\beta \mathcal{A}}+1}{e^{\beta \mathcal{A}}-1} \geq 2 k_{B} T,
$$

and $\lim _{\mathcal{A} \rightarrow 0, \delta \rightarrow \infty} Q=2 k_{B} T$. The two limiting scenarios at which $Q$ approaches $2 k_{B} T$ can be seen in Fig. S1E.

\section{The suboptimal condition of reversible Michaelis-Menten reactions}

We provide conditions at which $Q$ has a local minimum with respect to substrate concentration $([S])$ in reversible MM 
A

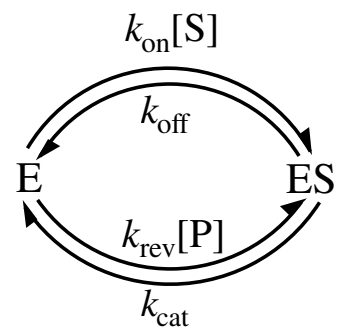

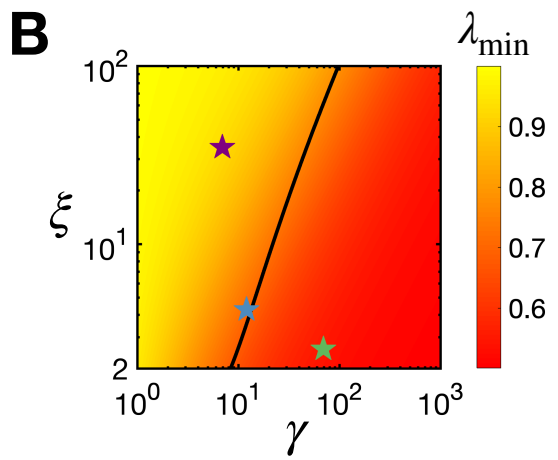

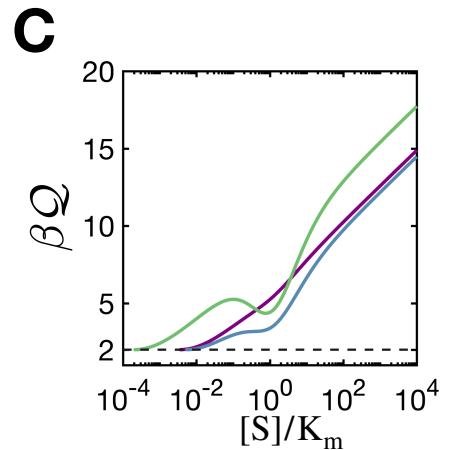

FIG. S2. Michaelis-menten type enzyme kinetics. (A) Schematic of MM type enzyme kinetics. (B) The minimum Fano factor with respect to $[\mathrm{S}]\left(\lambda_{\mathrm{min}}\right)$ plotted against dimensionless constants $\gamma=\frac{k_{\mathrm{cat}}}{\sqrt{k_{\mathrm{off}} k_{\mathrm{rev}}[\mathrm{P}]}}$ and $\xi=\frac{k_{\mathrm{off}}+k_{\mathrm{rev}}[\mathrm{P}]}{\sqrt{k_{\mathrm{off}} k_{\mathrm{rev}}[\mathrm{P}]}}$. To the left (resp. right) of the black curve, $Q$ is monotonic (resp. non-monotonic). (C) $Q$ plotted as a function of [S] normalized by $K_{m}=\frac{k_{\text {off }}+k_{\text {cat }}}{k_{\text {on }}}$. The corresponding $\gamma$ and $\xi$ values are shown in (B) by the star symbols with the matching color. The dotted line demarcates the lower bound $Q=2 k_{B} T$. The kinetic rate constants used for the plots are as follows: Green: $k_{\text {on }}=10^{8} \mathrm{M}^{-1} s^{-1}, k_{\text {off }}=30 s^{-1}, k_{\text {cat }}=10^{3} s^{-1}, k_{\text {rev }}[\mathrm{P}]=6.9 s^{-1}$; Blue: $k_{\text {on }}=10^{8} \mathrm{M}^{-1} s^{-1}, k_{\text {off }}=10 s^{-1}, k_{\text {cat }}=30 s^{-1}$, $k_{\text {rev }}[\mathrm{P}]=6.2 \times 10^{-1} s^{-1}$; Purple: $k_{\text {on }}=10^{8} \mathrm{M}^{-1} s^{-1}, k_{\text {off }}=50 s^{-1}, k_{\text {cat }}=10 s^{-1}, k_{\text {rev }}[\mathrm{P}]=4.1 \times 10^{-2} s^{-1}$.

reactions shown in Fig. S2A. First, the affinity $(\mathcal{A})$,

$$
\beta \mathcal{A}([S])=\ln \left(\frac{k_{\mathrm{on}} k_{\mathrm{cat}}[S]}{k_{\mathrm{off}} k_{\mathrm{rev}}[P]}\right)
$$

is a strictly increasing function of [S]. Next, the Fano factor $(\lambda)$ as a function of $[\mathrm{S}]$ is given by

$$
\begin{aligned}
\lambda([S]) & =\frac{k_{\mathrm{on}} k_{\mathrm{cat}}[S]+k_{\mathrm{off}} k_{\mathrm{rev}}[P]-2\left(\frac{k_{\mathrm{on}} k_{\mathrm{cat}}[S]-k_{\mathrm{off}} k_{\mathrm{rev}}[P]}{k_{\mathrm{on}}[S]+k_{\mathrm{off}}+k_{\mathrm{cat}}+k_{\mathrm{rev}}[P]}\right)^{2}}{k_{\mathrm{on}} k_{\mathrm{cat}}[S]-k_{\mathrm{off}} k_{\mathrm{rev}}[P]} \\
& =\frac{e^{\beta \mathcal{A}}+1}{e^{\beta \mathcal{A}}-1}-2 \frac{\gamma^{2}\left(e^{\beta \mathcal{A}}-1\right)}{\left(\gamma^{2}+\gamma \xi+e^{\beta \mathcal{A}}\right)^{2}},
\end{aligned}
$$

where $\gamma=\frac{k_{\text {cat }}}{\sqrt{k_{\text {off }} k_{\text {rev }}[P]}}$ and $\xi=\frac{k_{\text {off }}+k_{\text {rev }}[P]}{\sqrt{k_{\text {off }} k_{\text {rev }}[P]}}$ are dimensionless constants. $\lambda$ has a local minimum only when $\gamma>1$, or equivalently, when $k_{\text {cat }}>\sqrt{k_{\text {off }} k_{\text {rev }}[P]}$. At the limit of a strongly driven catalytic step $\left(k_{\mathrm{cat}} \gg k_{\mathrm{rev}}[P]\right)$, the expression for $\lambda$ simplifies to

$$
\lambda([S]) \approx 1-\frac{2 k_{\mathrm{cat}}[S]}{k_{\mathrm{on}}\left([S]+K_{\mathrm{m}}\right)^{2}},
$$

which is minimized at $[S]=K_{m}$ with $K_{m}=\left(k_{\text {off }}+k_{\text {cat }}\right) / k_{\text {on }}$ (Fig. $\mathrm{S2}$ B).

Since $\mathcal{A}$ is monotonic with $[\mathrm{S}]$, a local minimum of $Q([\mathrm{~S}])=\mathcal{A}([\mathrm{S}]) \lambda([\mathrm{S}])$ can only occur near to that of $\lambda([\mathrm{S}])$. Using this, we numerically determined the range of $\gamma$ and $\xi$ values at which $Q([\mathrm{~S}])$ has a local minimum away from the DB condition. For any $\xi$, there exists a $\gamma$ above which $Q[S]$ is non-monotonic (Fig. $\left[\mathrm{S} 2 \mathrm{~B}\right.$ ). Thus, when $k_{\mathrm{cat}} \gg k_{\mathrm{rev}}[P]$ and $k_{\text {cat }}$ is sufficiently larger than $\sqrt{k_{\text {off }} k_{\text {rev }}[P]}, Q([S])$ has a local minimum around $[\mathrm{S}] \approx K_{m}$.

\section{Mathematical expressions of $\eta$ and $\mathcal{A}$ for general copying processes}

Here, we provide the details of obtaining $\eta$ and $Q$ in the main text. A more mathematically rigorous treatment on the subject can be found in Ref. [37].

In the following, without loss of generality, we will define the error probability $(\eta)$ and affinity $(\mathcal{A})$ of copy processes by referring to the kinetic mechanism of mRNA translation. To begin, consider the ribosome at position $l$ of the mRNA sequence, decoding a specific codon type. For each amino-acid type $a$, there exists a set $T_{a}^{l}$, of associated aatRNAs, each of which represents a separate kinetic path of incorporating $a$ into the protein. If the codon at position $l$ is GGG, amino-acids Gly, Ala, Arg, Glu, Trp, and Val can be polymerized, with the following set of associated tRNAs: $T_{\text {Gly }}^{l}=\left\{\mathrm{tRNA}_{1}^{\mathrm{Gly}}, \mathrm{tRNA}_{2}^{\mathrm{Gly}}, \mathrm{tRNA}_{3}^{\mathrm{Gly}}\right\}, T_{\text {Ala }}^{l}=\left\{\mathrm{tRNA}_{1 \mathrm{~B}}^{\mathrm{Ala}}\right\}$, $T_{\text {Arg }}^{l}=\left\{\mathrm{tRNA}_{3}^{\mathrm{Arg}}, \mathrm{tRNA}_{5}^{\mathrm{Arg}}\right\}, T_{\mathrm{Glu}}^{l}=\left\{\mathrm{tRNA}_{2}^{\mathrm{Glu}}\right\}, T_{\text {Trp }}^{l}=$ $\left\{\mathrm{tRNA}^{\mathrm{Trp}}\right\}$, and $T_{\mathrm{Val}}^{l}=\left\{\mathrm{tRNA}_{1}^{\mathrm{Val}}\right\}$ (Fig. S7 .

At steady state, we assume that, $\eta_{a}^{l}$, the probability of incorporating amino-acid $a$ at position $l$, where the codon is specified, is given by

$$
\eta_{a}^{l}=\frac{\sum_{t \in T_{a}^{l}}\left\langle J_{a, t}^{l, \mathrm{pol}}\right\rangle}{\sum_{\alpha \in\{\mathrm{aa}\}} \sum_{t \in T_{\alpha}^{l}}\left\langle J_{\alpha, t}^{l, \mathrm{pol}}\right\rangle},
$$

where $\{\mathrm{aa}\}$ is the set of all amino-acids, and $\left\langle J_{a, t}^{l, \mathrm{pol}}\right\rangle$ is the polymerization current of aa-tRNA $t$.

Next, we define the affinity associated with polymerization using the previously discussed example of Gly incorporation at codon GGG. The polymerization affinity of Gly along the cognate kinetic path associated with Gly-tRNA $A_{1}^{\mathrm{Gly}}$ is

$$
\beta \mathcal{A}_{\mathrm{Gly}, \mathrm{Gly} 1}^{l, \mathrm{pol}}=-\beta \Delta \mu_{\mathrm{Gly}, \mathrm{Gly} 1}^{l, \mathrm{pol}}-\ln \left(\eta_{\mathrm{Gly}}^{l}\right),
$$


where $-\beta \Delta \mu_{\mathrm{Gly}, \mathrm{Gly} 1}^{l, \mathrm{pol}}=\ln \left(\frac{k_{\mathrm{on}}[\mathrm{C}] k_{\mathrm{rec}, f} k_{\mathrm{hyd}, f}^{\mathrm{C}} k_{\mathrm{pol}}^{\mathrm{C}}}{k_{\mathrm{off}} k_{\mathrm{rec}, r}^{\mathrm{C}} k_{\mathrm{hyd},}^{\mathrm{C}} k_{\mathrm{dep}}^{\mathrm{C}}}\right)$, and $[\mathrm{C}]$ represents the concentration of the ternary complex (Gly-tRNA ${ }_{1}^{\text {Gly }}$ )-(EF-Tu)-GTP (Fig. 3B). The term $\ln \left(\eta_{\mathrm{Gly}}^{l}\right)$ is required to account for the fact that Gly can be depolymerized at position $l$ only $\eta_{\mathrm{Gly}}^{l}$ fraction of the time. Similarly, the affinity of incorporating Gly along the near-cognate kinetic path associated with Gly-tRNA ${ }_{3}^{\text {Gly }}$ is

$$
\beta \mathcal{A}_{\mathrm{Gly}, \mathrm{Gly} 3}^{l, \mathrm{pol}}=-\beta \Delta \mu_{\mathrm{Gly}, \mathrm{Gly} 3}^{l, \mathrm{pol}}-\ln \left(\eta_{\mathrm{Gly}}^{l}\right),
$$

where $-\beta \Delta \mu_{\mathrm{Gly}, \mathrm{Gly} 3}^{l, \mathrm{pol}}=\ln \left(\frac{k_{\mathrm{on}}[\mathrm{NC}] k_{\mathrm{rec}, f} k_{\mathrm{hyd}, k_{\mathrm{pol}}^{\mathrm{NC}}}^{\mathrm{NC}}}{k_{\mathrm{off}} \mathrm{Nec}, r_{\mathrm{hyd}, r}^{\mathrm{NC}} k_{\mathrm{dep}}^{\mathrm{NC}}}\right)$, and $[\mathrm{NC}]=$ [(Gly-tRNA $\left.{ }_{3}^{\mathrm{Gly}}\right)$-(EF-Tu)-GTP]. Generally, we denote the polymerization affinity of amino-acid $a$ associated with aatRNA $t$ by $\mathcal{A}_{a, t}^{l, \mathrm{pol}}$.

Next, we let $\left\langle J_{a, t}^{l, \text { fut }}\right\rangle$ and $\Delta \mu_{a, t}^{l, \text { fut }}$ be the current and affinity of the futile cycle within the incorporation path of aa-tRNA $t$ associated with amino-acid $a$. Denoting the net polymerization flux by $\left\langle J^{l}\right\rangle\left(=\sum_{a \in\{a a\}} \sum_{t \in T_{a}^{l}}\left\langle J_{a, t}^{l, \text { pol}}\right\rangle\right)$, we can write the affinity of mRNA translation at position $l$ as

$\beta \mathcal{A l}^{l}=\underbrace{-\beta \sum_{a \in\{\mathrm{aa}\}} \sum_{t \in T_{a}^{l}}\left(\frac{\left\langle J_{a, t}^{l, \mathrm{pol}}\right\rangle}{\left\langle J^{l}\right\rangle} \Delta \mu_{a, t}^{l, \mathrm{pol}}+\frac{\left\langle J_{a, t}^{l, \mathrm{fut}}\right\rangle}{\left\langle J^{l}\right\rangle} \Delta \mu_{a, t}^{l, \mathrm{fut}}\right)}_{=-\beta \Delta \mu}-\underbrace{\sum_{a \in\{\mathrm{aa}\}} \eta_{a}^{l} \ln \eta_{a}^{l}}_{=I}$.

For the Bennett model, which involves two types of monomers, with one incorporation pathway each, without any futile cycles, we recover Eq. $\mathrm{S10}$ To estimate $I$ in the extended model of translation (Fig. 4), we sum the Shannonentropy of each position $l$ along the protein sequence.

\section{Computation of $\eta$ and $Q$}

We will work through the process of calculating $\eta$ and $Q$ in the T7 DNA polymerase model, for which we apply Koza's method of calculating currents and fluctuations in kinetic networks [71]. To simplify the notation, we will relabel the states in Fig. 2A by indices 1 through 5; i.e., $\mathrm{E}^{(1)} \rightarrow 1, c^{(2)} \rightarrow 2$, $c^{(3)} \rightarrow 3, i^{(2)} \rightarrow 4$, and $i^{(3)} \rightarrow 5$. Additionally, we will relabel the rate constants so that $k_{\mu, v}$ represents the rate of the reaction from state $\mu$ to $v$; i.e., $k_{1,2}=k_{\mathrm{on}}^{c}[\mathrm{dNTP}], k_{2,3}=k_{\mathrm{conf}}^{c}$, and so forth. Here, the depolymerization rate constants are set to $k_{1,3}=(1-\eta) k_{\mathrm{dep}}^{c}$ and $k_{1,5}=\eta k_{\mathrm{dep}}^{i}$.
To begin, we will compute the current of correct nucleotide incorporation $\left\langle J_{\mathrm{pol}}^{c}\right\rangle$. For the $i$-th chemical state $(i \in$ $\{1,2, \ldots, N\}$, where $N=5$ ) at time $t$, let $\mu(t) \equiv i+N \times n^{c}(t)$ be the generalized state of the system after completing $n^{c}(t)$ correct nucleotide incorporation cycles. Then, let $P(\mu, t)$ be the probability of the system to be in state $\mu$ at time $t$. The time evolution of $P(\mu, t)$ is given by

$$
\frac{\partial P(\mu, t)}{\partial t}=\sum_{\xi}\left[k_{\mu-\xi, \mu} P(\mu-\xi, t)-k_{\mu, \mu-\xi} P(\mu, t)\right],
$$

where the index $\xi$ runs through all states one reaction away from state $\mu$. Here, the periodicity of the network model constrains the rate constants so that $k_{\mu, v}=k_{i, j}$ for $\mu=i(\bmod N)$ and $v=j(\bmod N)$. Following Ref. [71], we define $P_{j}(\mu, t)$ as

$$
P_{j}(\mu, t) \equiv P(\mu, t) \delta_{\mu, j}^{N},
$$

where

$$
\delta_{\mu, j}^{N}= \begin{cases}1, & \text { if } j=\mu \quad(\bmod N) \\ 0, & \text { otherwise },\end{cases}
$$

for $j \in\{1,2, \ldots, N\}$. By multiplying $\delta_{\mu, j}^{N}$ to both sides of Eq. S22 and using the equality $\delta_{\mu, j}^{N}=\delta_{\mu-\xi, j-\xi}^{N}$, we get

$$
\frac{\partial P_{j}(\mu, t)}{\partial t}=\sum_{\xi}\left[k_{j-\xi, j} P_{j-\xi}(\mu-\xi, t)-k_{j, j-\xi} P_{j}(\mu, t)\right] \text {. }
$$

To derive the expression of $\left\langle J_{\text {pol }}^{c}\right\rangle$ as a function of the rate constants, we define the generating function

$$
\mathcal{G}_{j}^{c}(z, t) \equiv \sum_{\mu=-\infty}^{\infty} e^{z X_{\mu}} P_{j}(\mu, t),
$$

where $X_{\mu}$ is the coordinate for the correct nucleotide incorporation cycle at state $\mu$. Then, the time derivative of the generating function can be written as

$$
\frac{\partial \mathcal{G}_{j}^{c}(z, t)}{\partial t}=\sum_{i=1}^{N} \Gamma_{i, j}^{c}(z) \mathcal{G}_{i}^{c}(z, t)
$$

where the matrix $\Gamma^{c}(z)$ is defined as

$$
\Gamma_{i, j}^{c}(z)= \begin{cases}k_{i, j} e^{z d_{i, j},}, & \text { if } i \neq j \\ -\sum_{m=1(\neq i)}^{N} k_{i, m}, & \text { if } i=j\end{cases}
$$

and also shown in the matrix form below 


$$
\left[\begin{array}{ccccc}
-\left(k_{\mathrm{on}}^{c}[\mathrm{dNTP}]+k_{\mathrm{on}}^{i}[\mathrm{dNTP}]+k_{\mathrm{dep}}^{c}(1-\eta)+k_{\mathrm{dp}}^{i} \eta\right) & k_{\mathrm{on}}^{c}[\mathrm{dNTP}] & k_{\mathrm{dep}}^{c}(1-\eta) e^{-z} & k_{\mathrm{on}}^{i}[\mathrm{dNTP}] & k_{\mathrm{dep}}^{i} \eta \\
k_{\mathrm{off}}^{c} & -\left(k_{\mathrm{off}}^{c}+k_{\mathrm{conf}, f}^{c}\right) & k_{\mathrm{conf}, f}^{c} & 0 & 0 \\
k_{\mathrm{pol}}^{c} e^{z} & k_{\mathrm{conf}, r}^{c} & -\left(k_{\mathrm{pol}}^{c}+k_{\mathrm{conf}, r}^{c}\right) & 0 & 0 \\
k_{\mathrm{off}}^{i} & 0 & 0 & -\left(k_{\mathrm{off}}^{i}+k_{\mathrm{conf}, f}^{i}\right) & k_{\mathrm{conf}, f}^{i} \\
k_{\mathrm{pol}}^{i} & 0 & 0 & k_{\mathrm{con}, r}^{i} & -\left(k_{\mathrm{pol}}^{i}+k_{\mathrm{conf}, r}^{i}\right)
\end{array}\right]
$$

For the computation of $\left\langle J_{\mathrm{pol}}^{c}\right\rangle, d_{i, j}$ is defined as

$$
d_{i, j}= \begin{cases}1, & \text { if } i=3 \text { and } j=1 \\ -1, & \text { if } i=1 \text { and } j=3 \\ 0, & \text { otherwise. }\end{cases}
$$

Next, we define $\mathcal{G}^{c}(z, t) \equiv \sum_{i=1}^{N} \mathcal{G}_{i}^{c}(z, t)$ and denote the coordinate of the correct incorporation cycle by $X^{c}(t)$. Then, it can be shown that

$$
\left\langle J_{\mathrm{pol}}^{c}\right\rangle=\lim _{t \rightarrow \infty} \frac{\left\langle X^{c}(t)\right\rangle}{t}=\lim _{t \rightarrow \infty} \frac{\left.\partial_{z} \mathcal{G}^{c}(z, t)\right|_{z=0}}{t}=\left.\partial_{z} \Lambda_{0}^{c}(z)\right|_{z=0},
$$

and

$$
\begin{aligned}
\left\langle\left(\delta J_{\mathrm{pol}}^{c}\right)^{2}\right\rangle & =\lim _{t \rightarrow \infty} \frac{\left\langle\left(X^{c}(t)\right)^{2}\right\rangle-\left\langle X^{c}(t)\right\rangle^{2}}{t} \\
& =\lim _{t \rightarrow \infty} \frac{\left.\partial_{z}^{2} \mathcal{G}^{c}(z, t)\right|_{z=0}-\left(\left.\partial_{z} \mathcal{G}^{c}(z, t)\right|_{z=0}\right)^{2}}{t} \\
& =\left.\partial_{z}^{2} \Lambda_{0}^{c}(z)\right|_{z=0},
\end{aligned}
$$

where $\Lambda_{0}^{c}(z)$ denotes the maximum eigenvalue of the matrix $\Gamma^{c}(z)$. Now, let $C_{n}(z)$ denote the coefficients of the characteristic polynomial of $\Gamma^{c}(z)$ (i.e., $\sum_{n=0}^{N} C_{n}(z) \Lambda_{0}^{c}(z)^{n}=0$ ). Then, we can write the following expressions for $\left\langle J_{\text {pol }}^{c}\right\rangle$ and $\left\langle\left(\delta J_{\text {pol }}^{c}\right)^{2}\right\rangle$,

$$
\begin{aligned}
\left\langle J_{\mathrm{pol}}^{c}\right\rangle & =\left(\Lambda_{0}^{c}\right)^{\prime}=-\frac{C_{0}^{\prime}}{C_{1}}, \\
\left\langle\left(\delta J_{\mathrm{pol}}^{c}\right)^{2}\right\rangle & =\left(\Lambda_{0}^{c}\right)^{\prime \prime}=-\frac{C_{0}^{\prime \prime}+2 C_{1}^{\prime}\left(\Lambda_{0}^{c}\right)^{\prime}+2 C_{2}\left(\left(\Lambda_{0}^{c}\right)^{\prime}\right)^{2}}{C_{1}} .
\end{aligned}
$$

where, denotes the derivative with respect to $z$ evaluated at $z=0$, and $C_{1}$ and $C_{2}$ are evaluated at $z=0$. We can analogously compute the current of incorrect monomer incorporation, $\left\langle J_{\mathrm{pol}}^{i}\right\rangle$, by constructing the corresponding matrix $\Gamma^{i}(z)$, in which the non-diagonal entries of $k_{5,1}$ and $k_{1,5}$ are multiplied by $e^{z}$ and $e^{-z}$, respectively. Since $k_{1,3}$ and $k_{1,5}$ are functions of $\eta,\left\langle J_{\text {pol }}^{c}\right\rangle$ and $\left\langle J_{\text {pol }}^{i}\right\rangle$ are functions of $\eta$. Thus, we can solve for $\eta$ by the equality

$$
\eta=\frac{\left\langle J_{\mathrm{pol}}^{i}\right\rangle}{\left\langle J_{\mathrm{pol}}^{i}\right\rangle+\left\langle J_{\mathrm{pol}}^{c}\right\rangle} .
$$

After we obtain the numerical expression of $\eta$, we can construct the matrix $\Gamma(z)$ to calculate the total flux $\left\langle J_{\mathrm{pol}}\right\rangle=$ $\left\langle J_{\text {pol }}^{c}\right\rangle+\left\langle J_{\text {pol }}^{i}\right\rangle$ and its fluctuation $\left\langle\left(\delta J_{\text {pol }}\right)^{2}\right\rangle$. With known values of $\eta$ and $\left\langle J_{\text {pol }}\right\rangle$, the affinity of replication $(\mathcal{A})$ can be computed by Eq. S21] In sum, we have demonstrated how to calculate $Q=\mathcal{A}\left\langle\left(\delta J_{\mathrm{pol}}\right)^{2}\right\rangle /\left\langle J_{\mathrm{pol}}\right\rangle$ for the T7 DNA polymerase model.

We can calculate $\eta$ and $Q$ of the simplified ribosome model in a similar way. To obtain $\left\langle J_{\text {pol }}^{\mathrm{C}}\right\rangle$ as a function of $\eta$, we construct the corresponding matrix $\Gamma^{\mathrm{C}}(z)$, in which we multiply the non-diagonal entries corresponding to $k_{\mathrm{pol}, f}^{\mathrm{C}}$ and $k_{\mathrm{pol}, r}^{\mathrm{C}}$ by $e^{z}$ and $e^{-z}$, respectively. The expression for $\left\langle J_{\mathrm{pol}}^{\mathrm{NC}}\right\rangle$ is obtained analogously, by constructing the corresponding matrix $\Gamma^{\mathrm{NC}}(z)$. After solving for the numerical value of the error probability, we can calculate the total polymerization rate $\left\langle J_{\mathrm{pol}}\right\rangle$ and its fluctuation $\left\langle\left(\delta J_{\mathrm{pol}}\right)^{2}\right\rangle$ by constructing the corresponding matrix $\Gamma(z)$. When computing the affinity, we must also include the contribution from the futile cycle fluxes. To calculate the futile cycle current $\left\langle J_{\text {fut }}\right\rangle$, we construct the corresponding matrix $\Gamma^{\mathrm{fut}}(z)$, in which we multiply the non-diagonal entries corresponding to $k_{\mathrm{PR}, f}^{\mathrm{C}}, k_{\mathrm{PR}, f}^{\mathrm{NC}}, k_{\mathrm{PR}, r}^{\mathrm{C}}$ and $k_{\mathrm{PR}, r}^{\mathrm{NC}}$ by $e^{z}, e^{z}, e^{-z}$ and $e^{-z}$, respectively. Finally, the affinity of translation can be computed by Eq.S21

\section{Stochastic simulation of DNA replication}

We simulated the replication of the first 300 base pairs of the T7 DNA polymerase gene sequence at the single molecule level, with Gillespie's algorithm [72]. The simulation starts with the polymerase in the apo state at the 5' end of the gene sequence. The only reactions available at this state are the binding reactions of the 4 dNTPs, which are assumed to be at equal concentrations. After the binding of a dNTP, the simulation trajectories are generated based on the kinetic network shown in Fig. 2A. After each polymerization reaction, the polymerase translocates on the DNA and reads the next nucleotide. The simulation is terminated when the $300^{\text {th }}$ nucleotide of the gene sequence is polymerized.

The dynamics of DNA replication simulations are studied using the ensemble of trajectories generated. The total number of steps $\left(N_{\text {rep }}\right)$ in completing the replication of the DNA sequence varies from one realization to another. Selecting the completion time of replication $(\mathcal{T})$ as the output observable for each realization, we define TUR of replication as

$$
Q=\left[-\Delta \mu+\beta^{-1} I\right] \frac{\left\langle(\delta \mathcal{T})^{2}\right\rangle}{\langle\mathcal{T}\rangle^{2}},
$$

where the dissipation has contributions from the free energy 
drive $(\Delta \mu)$ and Shannon-entropy $(I)$. Denoting the forward and reverse rate constants of each kinetic step by $k_{i, f}$ and $k_{i, r}$ for $i=1, \ldots, N_{\text {rep }}$, we can compute the average free energy drive by $-\beta \Delta \mu=\left\langle\sum_{i=1}^{N_{r e p}} \ln \frac{k_{i, f}}{k_{i, r}}\right\rangle$, where $\langle\ldots\rangle$ denotes the average over the ensemble of $10^{4}$ realizations. The entropic contribution is computed as $I=-\sum_{l=1}^{300} \sum_{i_{\mathrm{dNTP}}=1}^{4} \eta_{i_{\mathrm{dNTP}}}^{l} \ln \eta_{i_{\mathrm{dNTP}}}^{l}$, where $\eta_{i_{\mathrm{dNTP}}}^{l}$ is the probability of incorporating one of the 4 types of dNTPs, at the $l$-th position.

\section{The ribosome model}

We provide more details on the model of translation by the ribosome in Fig. 3 Our model is a modified version of that from Rudorf et. al. [73], in which we combine all linear chains of consecutive and irreversible reactions into single reactions. For instance, consider two consecutive and irreversible reactions $(1) \rightarrow(2)$ and $(2) \rightarrow(3)$, with respective rate constants $k_{12}$ and $k_{23}$, defined among three states (1), (2) and (3). If there are no other reactions associated with the state (2), we remove the state (2), and define a new reaction $(1) \rightarrow$ (3) with the rate constant $k_{13}^{-1} \equiv k_{12}^{-1}+k_{23}^{-1}$.

To calculate the entropy productions, we defined reverse rate constants for all reactions, which were constrained by the affinity of the corresponding kinetic cycle. We assumed that affinities of the parallel kinetic cycles for the cognate and nearcognate aa-tRNAs were identical. The affinity of the futile cycle $\left(\Delta \mu_{\text {fut }}\right)$ comes from GTP hydrolysis, which dissipates $\approx$ $20 k_{B} T$ [74]. Therefore, we applied the following constraints, where $-\beta \Delta \mu_{\text {fut }}=20$.

$$
\begin{array}{r}
\ln \left(\frac{k_{\mathrm{on}}[\mathrm{C}] k_{\mathrm{rec}, f} k_{\mathrm{hyd}, f}^{\mathrm{C}} k_{\mathrm{PR}, f}^{\mathrm{C}}}{k_{\mathrm{off}} k_{\mathrm{rec}, r}^{\mathrm{C}} k_{\mathrm{hyd}, r}^{\mathrm{C}} k_{\mathrm{PR}, r}^{\mathrm{C}}\left[\mathrm{C}^{\prime}\right]}\right)=-\beta \Delta \mu_{\mathrm{fut}}, \\
\ln \left(\frac{k_{\mathrm{on}}[\mathrm{NC}] k_{\mathrm{rec}, f} k_{\mathrm{hyd}, f}^{\mathrm{NC}} k_{\mathrm{PR}, f}^{\mathrm{NC}}}{k_{\mathrm{off}} k_{\mathrm{rec}, r}^{\mathrm{NC}} k_{\mathrm{hyd}, r}^{\mathrm{NC}} k_{\mathrm{PR}, r}^{\mathrm{NC}}\left[\mathrm{NC}^{\prime}\right]}\right)=-\beta \Delta \mu_{\mathrm{fut}} .
\end{array}
$$

The affinity involved with polymerization $\left(\Delta \mu_{\mathrm{pol}}\right)$ can be estimated as the sum of the free energies of GTP hydrolysis, peptide bond formation, and the cleavage of the ester bond between the tRNA and the amino-acid. The hydrolysis of the GTP molecule incurs the dissipation of $\approx 20 k_{B} T$. Conversely, each peptide bond synthesized stores $\approx 5 k_{B} T$ of free energy [75]. The standard free energy of the ester bond between the amino-acid and the tRNA is $\approx 12 k_{B} T[74,76]$. Since the ratio of charged to uncharged tRNAs is $\approx 10$ fold [77], the net free energy of the ester bond between the amino-acid and tRNA is $\approx 15 k_{B} T$. Then, $-\beta \Delta \mu_{\text {pol }} \approx 30$, which gives the following constraints

$$
\begin{aligned}
\ln \left(\frac{k_{\mathrm{on}}[\mathrm{C}] k_{\mathrm{rec}, f} k_{\mathrm{hyd}, f}^{\mathrm{C}} k_{\mathrm{pol}}^{\mathrm{C}}}{k_{\mathrm{off}} k_{\mathrm{rec}, r}^{\mathrm{C}} k_{\mathrm{hyd}, r}^{\mathrm{C}} k_{\mathrm{dep}}^{\mathrm{C}}}\right) & =-\beta \Delta \mu_{\mathrm{pol}}, \\
\ln \left(\frac{k_{\mathrm{on}}[\mathrm{NC}] k_{\mathrm{rec}, f} k_{\mathrm{hyd}, f}^{\mathrm{NC}} k_{\mathrm{pol}}^{\mathrm{NC}}}{k_{\mathrm{off}} k_{\mathrm{rec}, r}^{\mathrm{NC}} k_{\mathrm{hyd}, r}^{\mathrm{NC}} k_{\mathrm{dep}}^{\mathrm{NC}}}\right) & =-\beta \Delta \mu_{\mathrm{pol}} .
\end{aligned}
$$

The terms $k_{\text {dep }}^{\mathrm{C}}$ and $k_{\mathrm{dep}}^{\mathrm{NC}}$ implicitly take into account the concentration of tRNA and (EF-Tu)-GDP, which detach during the final polymerization step. In order to fully constrain all the rate constants, we set $k_{\mathrm{hyd}, r}^{\mathrm{C}}=10^{-3} k_{\mathrm{hyd}, f}^{\mathrm{C}}$ and $k_{\mathrm{hyd}, r}^{\mathrm{NC}}=$ $10^{-3} k_{\mathrm{hvd} . f}^{\mathrm{NC}}$. Modest changes to these affinity related constraints (Eqs S35 S38) do not affect the qualitative conclusions of our work.

\section{Ternary complex concentration}

The ternary complex concentration was modeled as a function of the concentration of its components, aa-tRNA, EF-Tu, GTP, and GDP. First, EF-Tu binds with GTP and GDP to form (EF-Tu)-GTP and (EF-Tu)-GDP, respectively. Then, aatRNA binds with (EF-Tu)-GTP and (EF-Tu)-GDP to form (aatRNA)-(EF-Tu)-GTP and (aa-tRNA)-(EF-Tu)-GDP, respectively [78]. Here, (aa-tRNA)-(EF-Tu)-GTP and (aa-tRNA)(EF-Tu)-GDP represent the combined total of all the respective 42 individual ternary complexes. Assuming equilibrium, we can write the following equalities,

$$
\begin{aligned}
{[(\text { aa-tRNA })-(\mathrm{EF}-\mathrm{Tu})-\mathrm{GTP}] } & =\frac{[\mathrm{aa}-\mathrm{tRNA}][(\mathrm{EF}-\mathrm{Tu})-\mathrm{GTP}]}{K_{\mathrm{aaGTP}}}, \\
{[(\mathrm{aa}-\mathrm{tRNA})-(\mathrm{EF}-\mathrm{Tu})-\mathrm{GDP}] } & =\frac{[\mathrm{aa}-\mathrm{tRNA}][(\mathrm{EF}-\mathrm{Tu})-\mathrm{GDP}]}{K_{\mathrm{aaGDP}}}, \\
{[(\mathrm{EF}-\mathrm{Tu})-(\mathrm{GTP})] } & =\frac{[\mathrm{EF}-\mathrm{Tu}][\mathrm{GTP}]}{K_{\mathrm{GTP}}}, \\
{[(\mathrm{EF}-\mathrm{Tu})-(\mathrm{GDP})] } & =\frac{[\mathrm{EF}-\mathrm{Tu}][\mathrm{GDP}]}{K_{\mathrm{GDP}}},
\end{aligned}
$$

where the respective dissociation constants are set to $K_{\text {aaGTP }}=10^{-1} \mu \mathrm{M}$ [78], $K_{\text {aaGDP }}=14 \mu \mathrm{M}$ [78], $K_{\mathrm{GTP}}=$ $6 \times 10^{-2} \mu M$ [79], and $K_{\mathrm{GDP}}=10^{-3} \mu \mathrm{M}$ [79]. Using Eqs. S39. S42, we can solve for the concentration of all chemical species given the total [EF-Tu], [aa-tRNA], [GTP], and [GDP]. Unless specified otherwise, all the ribosome model plots (Figs. 3 and 4 are made at the cellular condition, with [EF-Tu] $=250 \mu \mathrm{M}$ [51], [aa-tRNA] $\approx 200 \mu \mathrm{M}$ [49], [GTP] $=$ $5000 \mu \mathrm{M}$ [80], and [GDP] $=700 \mu \mathrm{M}$ [80]. At the cellular condition, the concentration of the GTP bound ternary complex is [(aa-tRNA)-(EF-Tu)-GTP] $\approx$ [aa-tRNA] $\approx 200 \mu \mathrm{M}$.

Assuming that all species of aa-tRNA were bound to (EFTu)-GTP and (EF-Tu)-GDP with equal binding constants $K_{\text {aaGTP }}$ and $K_{\text {aaGDP }}$, we computed the concentration of individual ternary complexes by referencing the measured concentration ratios among individual aa-tRNA species. For instance, the concentration of the cognate ternary complexes of codon UUU, which encodes for Phe, are

$$
\begin{aligned}
{[C] } & =\frac{\left[\text { Phe-tRNA }^{\text {Phe }}\right]_{\mathrm{WT}}}{[\text { aa-tRNA }]_{\mathrm{WT}}}[(\text { aa-tRNA })-(\text { EF-Tu })-G T P], \\
{\left[\mathrm{C}^{\prime}\right] } & =\frac{\left[\text { Phe-tRNA }{ }^{\text {Phe }}\right]_{\mathrm{WT}}}{[\text { aa-tRNA }]_{\mathrm{WT}}}[(\text { aa-tRNA)-(EF-Tu)-GDP }],
\end{aligned}
$$


where the subscript WT represents the cellular concentrations obtained from Ref. [49].
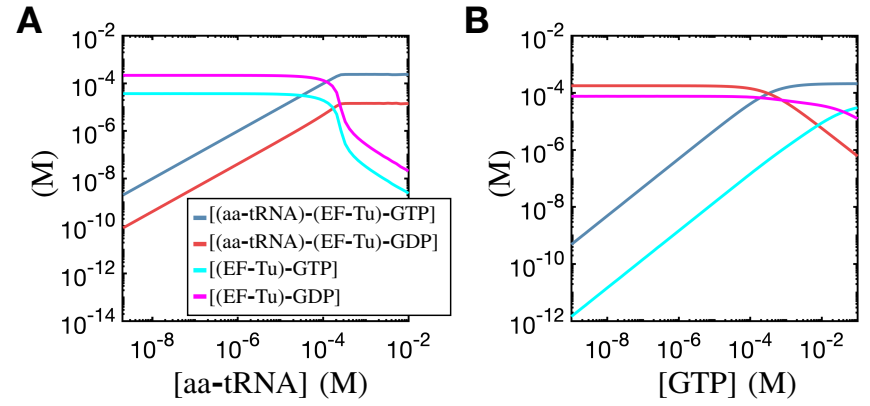

FIG. S3. The concentration of the components of the ternary complex as functions of [aa-tRNA] and [GTP]. (A) The dependence of [(aa-tRNA)-(EF-Tu)-GTP], [(aa-tRNA)-(EF-Tu)-GDP], [(EF-Tu)-GTP], and [(EF-Tu)-GDP] on [aa-tRNA], where [GTP] was fixed at $5 \mathrm{mM}$. (B) The dependence of [(aa-tRNA)-(EF-Tu)GTP], [(aa-tRNA)-(EF-Tu)-GDP], [(EF-Tu)-GTP], and [(EF-Tu)GDP] on [GTP], where [aa-tRNA] was fixed at $\approx 0.2 \mathrm{mM}$. The curves are color-coded identically as those in (A). For both plots, we assumed cellular levels of $[\mathrm{GDP}](=0.7 \mathrm{mM})$ and $[\mathrm{EF}-\mathrm{TU}](=0.25 \mathrm{mM})$, and used equilibrium dissociation constants as described in the SI text.

\section{The extended model of translation}

Excluding the three stop codons, there are 61 types of codons encoding for 20 amino-acids. For E. coli, the 43 types of tRNAs were identified by Dong et. al [49]. Out of these, the pairs Gly1-Gly2 and Ile1-Ile2 were not differentiated in the concentration measurements. In our simulations, we assumed that Gly1 and Gly2 (resp. Ile1 and Ile2) were each present in the cellular milieu at half of the measured concentration of the Gly1-Gly2 pair (resp. Ile1-Ile2 pair). We removed the seleno-cysteine carrying tRNA from the analysis, since it is low in concentration, and it is incorporated into the polypeptide through a different kinetic scheme from the rest of the aa-tRNAs. Overall, we included total 42 types of aatRNAs in the extended translation model, with measurements from $E$. coli dividing every $\approx 86$ minutes [49]. The cognate, near-cognate, and non-cognate groupings of aa-tRNAs for each codon is shown in Fig. S7.

mRNA translation by the ribosome at the single molecule level is simulated with Gillespie's algorithm [72]. The simulation starts with the ribosome in the apo state at the start codon. The only reactions available at this state are the bindings of the 42 aa-tRNAs, the concentrations of which were taken from Dong et. al. [49]. After the binding of an aatRNA, the simulation trajectories were generated on the kinetic network shown in Fig. 3B. After each polymerization reaction, the ribosome reads the next codon, translocating along the mRNA. The simulation is terminated when the ribosome completes the translation of the last codon.

\section{The Hopfield model}

We provide more details on the modified Hopfield model [1]. The reaction cycle of the Hopfield model is composed of substrate binding $(\mathbf{E}+\mathbf{C} \rightleftharpoons \mathbf{E C}$ and $\mathbf{E}+\mathbf{I} \rightleftharpoons \mathbf{E I})$, followed by the effectively irreversible steps of ATP hydrolysis $\left(\mathbf{E C} \rightleftharpoons \mathbf{E C}^{*}\right.$ and $\left.\mathbf{E I} \rightleftharpoons \mathbf{E I}^{*}\right)$ and polymerization $\left(\mathbf{E C}^{*} \rightleftharpoons \mathbf{E}\right.$ and $\mathbf{E I}{ }^{*} \rightleftharpoons \mathbf{E}$ ) (Fig. S4). At states $\mathbf{E C}^{*}$ and $\mathbf{E I}^{*}$, the substrate can also dissociate through the proofreading reaction (PR, $\mathbf{E C}^{*} \rightleftharpoons \mathbf{E}+\mathbf{C}$ and $\mathbf{E I}^{*} \rightleftharpoons \mathbf{E}+\mathbf{I}$ ), which is also effectively irreversible.

In the original formulation of the Hopfield model, the forward kinetic rate constants of correct and incorrect pathways were identical. In our modified version, the forward constants satisfy the following relations

$$
e^{\beta \delta}=\frac{k_{\mathrm{on}}^{c}}{k_{\mathrm{on}}^{i}}=\frac{k_{\mathrm{hyd}, f}^{c}}{k_{\mathrm{hyd}, f}^{i}}=\frac{k_{\mathrm{pol}}^{c}}{k_{\mathrm{pol}}^{i}}=\frac{k_{\mathrm{PR}, r}^{c}}{k_{\mathrm{PR}, r}^{i}},
$$

with $\beta \delta>0$. Next, to allow for error reduction by proofreading, we set the forward kinetic rates so that $k_{\text {pol }}^{c} \ll k_{\text {hyd }, f}^{c} \ll$ $k_{\mathrm{on}}^{c}$. Finally, we constrained the reverse reaction rates so that the affinities associated with the kinetic cycles are $\Delta \mu_{\mathrm{pol}}^{c}=$ $-20 k_{B} T$, and $\Delta \mu_{\mathrm{pol}}^{i}=\Delta \mu_{\text {fut }}^{c}=\Delta \mu_{\text {fut }}^{i}=-18 k_{B} T$. The rate constants used to generate Fig. 5 and Fig. 59 are provided in Table S3.

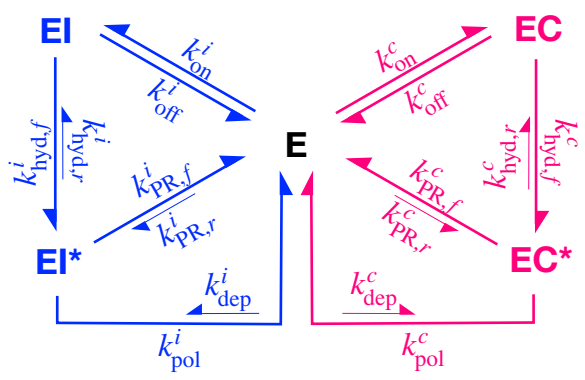

FIG. S4. Schematic of the modified Hopfield model [1]. 

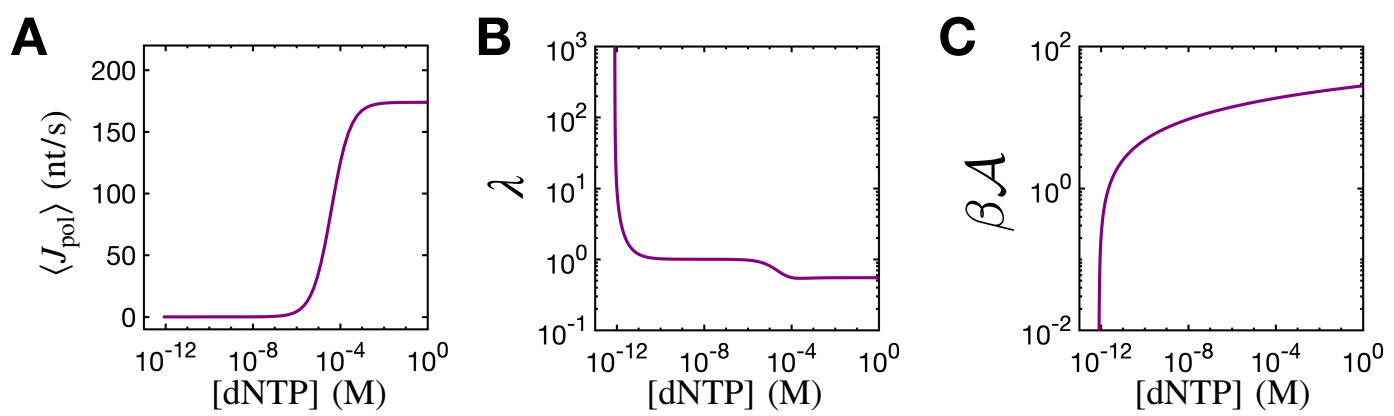

FIG. S5. Dynamical properties of the exonuclease-deficient T7 DNA polymerase, obtained at identical conditions as in Fig. 2 (A) The reaction current $\left(\left\langle J_{\text {pol }}\right\rangle\right)$, (B) Fano factor of the reaction current $(\lambda)$, and $(C)$ the affinity $(\mathcal{A})$ are plotted against [dNTP].
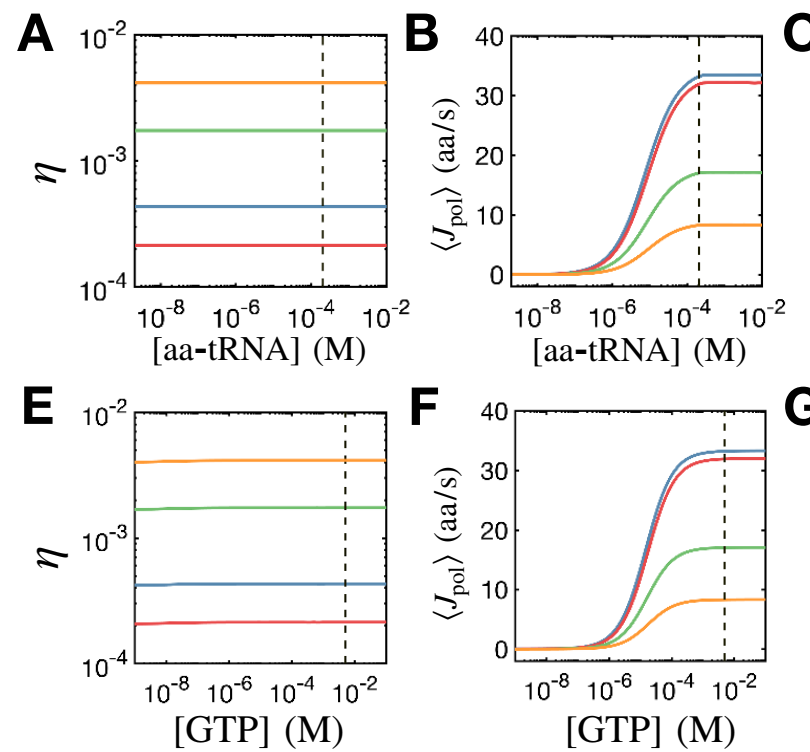
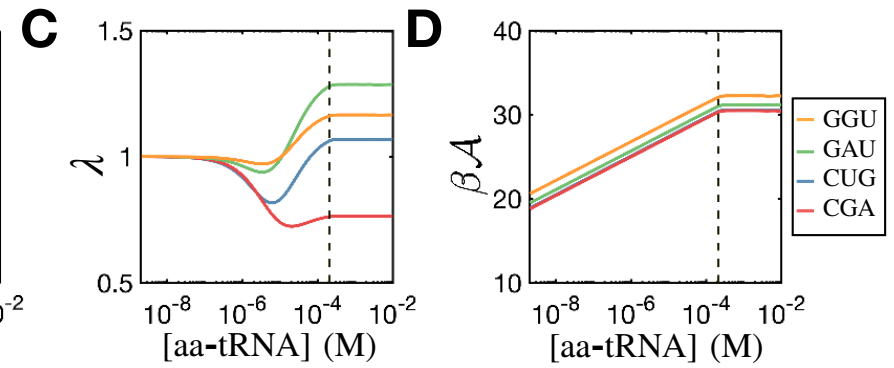

G

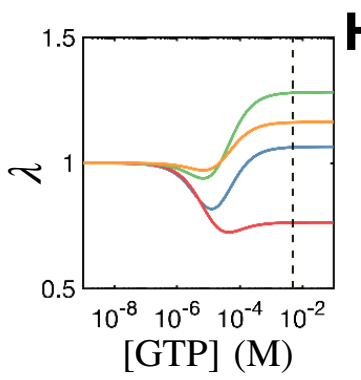

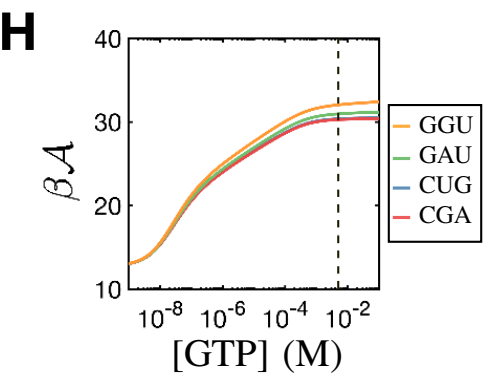

FIG. S6. Dynamical properties of mRNA translation by E. coli ribosome. (A) The error probability $(\eta)$, (B) polymerization current $\left(\left\langle J_{\text {pol }}\right\rangle\right)$, (C) Fano factor of the polymerization current $(\lambda)$, and (D) affinity $(\mathcal{A})$ plotted against [aa-tRNA], at identical conditions as in Fig. 3P. $(\mathrm{E})$ The error probability $(\eta),(\mathrm{F})$ polymerization current $\left(\left\langle J_{\mathrm{pol}}\right\rangle\right),(\mathrm{G})$ Fano factor of the polymerization current $(\lambda)$, and $(\mathrm{H})$ affinity $(\mathcal{A})$ plotted against [GTP], at identical conditions as in Fig. 3E. For all plots, the dashed black line represents the cellular condition in E. coli. 

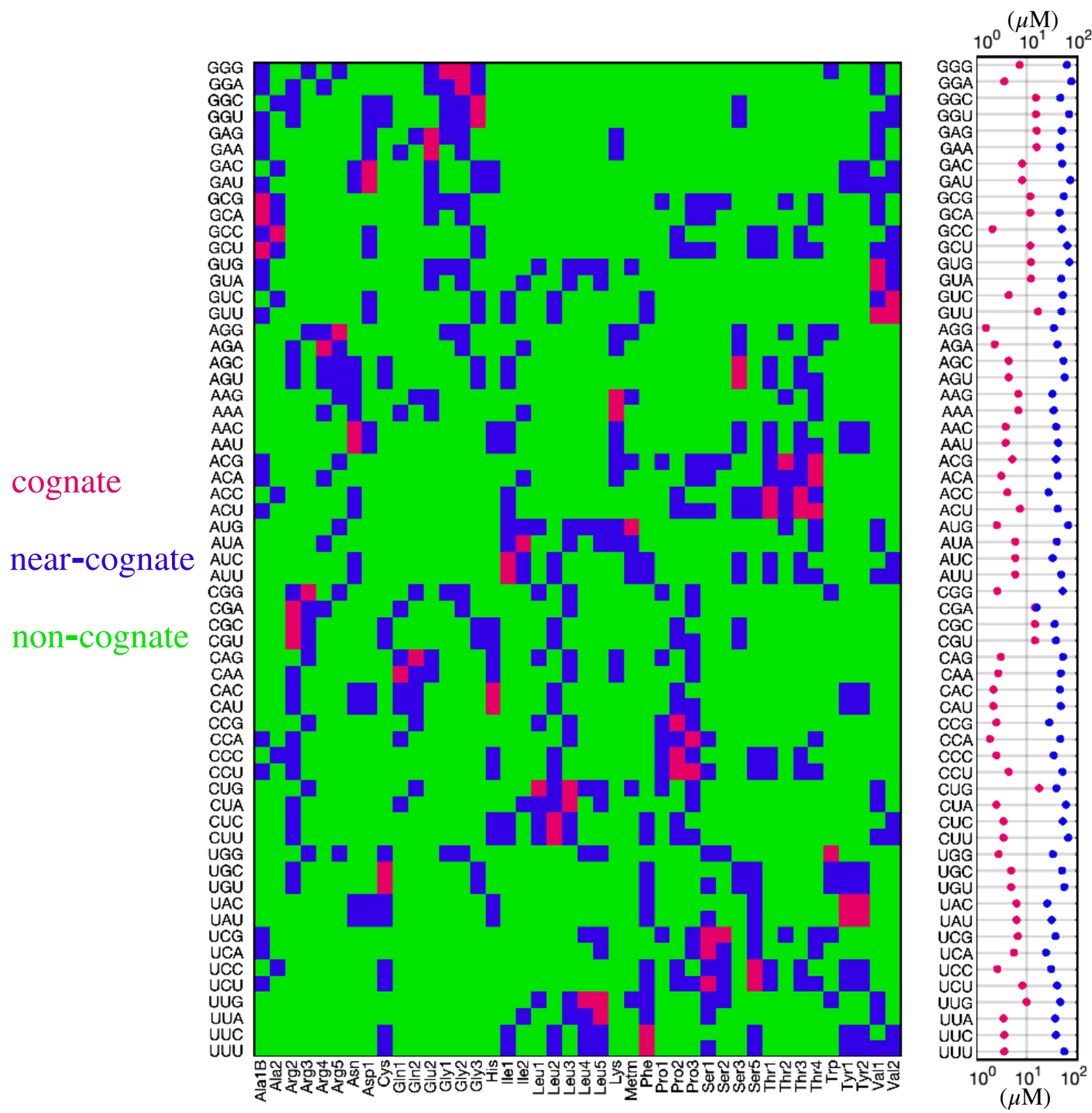

FIG. S7. (Left) The groupings of cognate (red), near-cognate (blue), and non-cognate (green) aa-tRNAs for each codon [73]. (Right) The sum of the concentration of the cognate (red) and near-cognate (blue) aa-tRNA species, plotted for each codon [49]. 

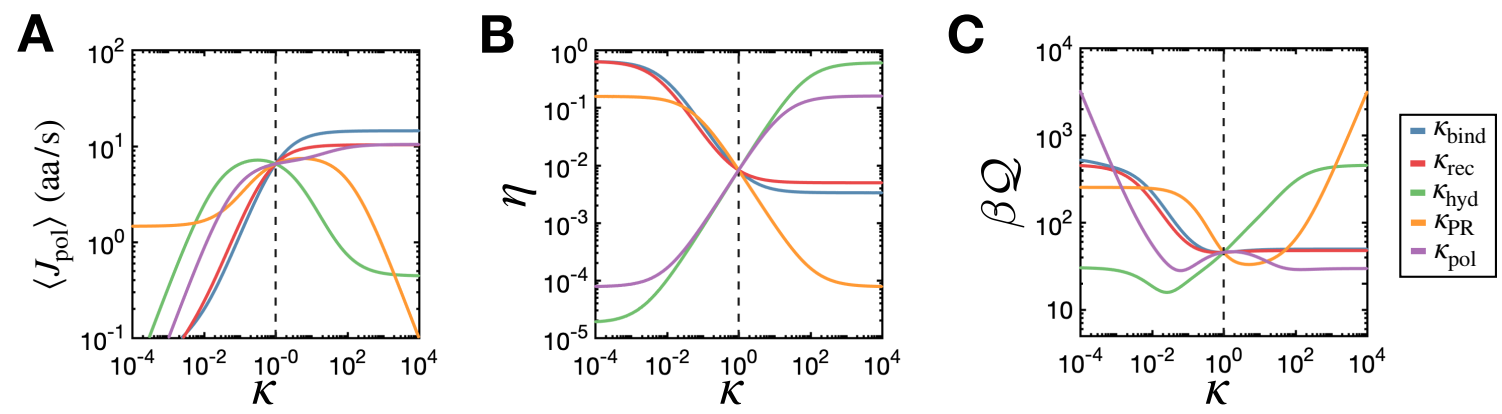

FIG. S8. Dynamical properties of mRNA translation by the E. coli ribosome with respect to perturbations to the wild type rate constants. (A) The polymerization current $\left(\left\langle J_{\mathrm{pol}}\right\rangle\right)$, (B) the error probability $(\eta)$, and (C) $Q$ as functions of $\kappa_{\text {bind }}, \kappa_{\mathrm{rec}}, \kappa_{\mathrm{hyd}}, \kappa_{\mathrm{PR}}$, and $\kappa_{\mathrm{pol}}$. The perturbative parameters $\kappa_{\text {bind }}, \kappa_{\text {rec }}, \kappa_{\text {hyd }}, \kappa_{\mathrm{PR}}$, and $\kappa_{\text {pol }}$ were each multiplied to the reactions associated with binding, codon-recognition, GTP-hydrolysis, proofreading, and polymerization, respectively. The black dashed lines represent the wild type condition for the codon UUU.
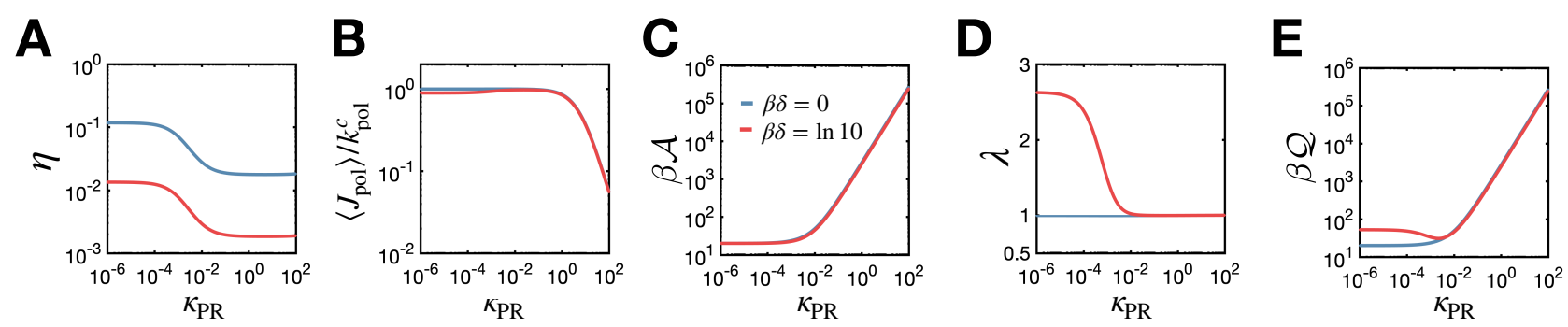

FIG. S9. Dynamical properties of the modified Hopfield model with kinetic discrimination. (A) The error probability $(\eta)$, (B) normalized polymerization current $\left(\left\langle J_{\mathrm{pol}}\right\rangle / k_{\mathrm{pol}}^{c}\right),(\mathrm{C})$ affinity $(\mathcal{A})$, (D) Fano factor $(\lambda)$, and (E) $Q$ as functions of $\kappa_{\mathrm{PR}}$. For (A)-(E) The perturbative parameter $\kappa_{\mathrm{PR}}$ is multiplied to the rates $k_{\mathrm{PR}, f}^{c}, k_{\mathrm{PR}, f}^{i}, k_{\mathrm{PR}, r}^{c}$ and $k_{\mathrm{PR}, r}^{i}$. The blue line presents the original Hopfield model with $\beta \delta=0$, and the orange line represents the modified Hopfield model with kinetic discrimination, with $\beta \delta=\ln 10$. In (C), the orange and blue lines are nearly identical. The rate constants used to make these plots are given in Table S3. 
TABLE S1. Rate constants for the T7 DNA polymerase, from Ref. [38]. The rate constants $k_{\mathrm{dep}}^{c}$ and $k_{\mathrm{dep}}^{i}$ are determined such that the affinities of correct and incorrect monomer incorporations are 20 and $15 k_{B} T$, respectively, when [dNTP] $=100 \mu \mathrm{M}$ [40, 81]. The terms $k_{\text {dep }}^{c}$ and $k_{\text {dep }}^{i}$ implicitly take into account the concentration of PPi which detaches during the final polymerization step.

\begin{tabular}{c|c}
\hline$k_{\mathrm{on}}^{c}$ & $10^{2} \mu \mathrm{M}^{-1} s^{-1}$ \\
\hline$k_{\mathrm{conf}, f}^{c}$ & $6.0 \times 10^{2} s^{-1}$ \\
\hline$k_{\mathrm{pol}}^{c}$ & $3.6 \times 10^{2} s^{-1}$ \\
\hline$k_{\mathrm{off}}^{c}$ & $2.8 \times 10^{3} s^{-1}$ \\
\hline$k_{\mathrm{conf}, r}^{c}$ & $1.6 s^{-1}$ \\
\hline$k_{\mathrm{dep}}^{c}$ & $10^{-3} s^{-1}$ \\
\hline$k_{\mathrm{on}}^{i}$ & $10^{2} \mu \mathrm{M}^{-1} s^{-1}$ \\
\hline$k_{\mathrm{conf}, f}^{i}$ & $2.2 \times 10^{2} s^{-1}$ \\
\hline$k_{\mathrm{pol}}^{i}$ & $3.0 \times 10^{-1} s^{-1}$ \\
\hline$k_{\mathrm{off}}^{i}$ & $2.0 \times 10^{4} s^{-1}$ \\
\hline$k_{\mathrm{conf}, r}^{i}$ & $4.2 \times 10^{2} s^{-1}$ \\
\hline$k_{\mathrm{dep}}^{i}$ & $2.4 \times 10^{-8} s^{-1}$ \\
\hline
\end{tabular}

TABLE S2. Rate constants for the wild type E. coli ribosome, from Ref. [73]. The rate constants $k_{\mathrm{PR}, r}^{\mathrm{C}}, k_{\mathrm{dep}}^{\mathrm{C}}, k_{\mathrm{PR}, r}^{\mathrm{NC}}$, and $k_{\mathrm{dep}}^{\mathrm{NC}}$ were determined from the constraints associated with the affinity of the kinetic cycles at the wild type condition (Eqs S35,S38).

\begin{tabular}{c|c}
\hline$k_{\mathrm{on}}$ & $9.4 \times 10 \mu \mathrm{M}^{-1} s^{-1}$ \\
\hline$k_{\mathrm{off}}$ & $1.4 \times 10^{3} s^{-1}$ \\
\hline$k_{\mathrm{rec}, f}$ & $2.1 \times 10^{3} s^{-1}$ \\
\hline$k_{\mathrm{rec}, r}^{\mathrm{C}}$ & $2 s^{-1}$ \\
\hline$k_{\mathrm{hyd}, f}^{\mathrm{C}}$ & $3.75 \times 10^{2} s^{-1}$ \\
\hline$k_{\mathrm{hyd}, r}^{\mathrm{C}}$ & $k_{\mathrm{hyd}, f}^{\mathrm{C}} \times 10^{-3}$ \\
\hline$k_{\mathrm{PR}, f}^{\mathrm{C}}$ & $1 s^{-1}$ \\
\hline$k_{\mathrm{pol}}^{\mathrm{C}}$ & $1.1 \times 10^{2} s^{-1}$ \\
\hline$k_{\mathrm{rec}, r}^{\mathrm{NC}}$ & $2.7 \times 10^{3} s^{-1}$ \\
\hline$k_{\mathrm{hyd}, f}^{\mathrm{NC}}$ & $4.9 s^{-1}$ \\
\hline$k_{\mathrm{hyd}, r}^{\mathrm{NC}}$ & $k_{\mathrm{hyd}, f}^{\mathrm{NC}} \times 10^{-3}$ \\
\hline$k_{\mathrm{PR}, f}^{\mathrm{NC}}$ & $6 s^{-1}$ \\
\hline$k_{\mathrm{pol}}^{\mathrm{NC}}$ & $2.7 \times 10^{-1} s^{-1}$ \\
\hline
\end{tabular}


TABLE S3. Rate constants for the Hopfield model with kinetic discrimination, where $-\beta \Delta \mu_{c}=2$ and $-\beta \Delta \mu_{i}=0$. The parameters $\delta$ and $\kappa_{\mathrm{PR}}$ are as defined in the main text.

\begin{tabular}{c|c}
\hline$k_{\mathrm{on}}^{c}$ & $10^{3} s^{-1}$ \\
\hline$k_{\mathrm{off}}^{c}$ & $k_{\mathrm{on}}^{c} e^{\beta \Delta \mu_{c}}$ \\
\hline$k_{\mathrm{hyd}, f}^{c}$ & $1 s^{-1}$ \\
\hline$k_{\mathrm{hyd}, r}^{c}$ & $k_{\mathrm{hyd}, f}^{c} e^{-10}$ \\
\hline$k_{\mathrm{pol}}^{c}$ & $10^{-3} s^{-1}$ \\
\hline$k_{\mathrm{dep}}^{c}$ & $k_{\mathrm{pol}}^{c} e^{-8}$ \\
\hline$k_{\mathrm{PR}, f}^{c}$ & $\kappa_{\mathrm{PR}} e^{\beta \Delta \mu_{c}} s^{-1}$ \\
\hline$k_{\mathrm{PR}, r}^{c}$ & $k_{\mathrm{PR}, f}^{c} e^{-8-\beta \Delta \mu_{c}}$ \\
\hline$k_{\mathrm{on}}^{i}$ & $k_{\mathrm{on}}^{c} e^{-\beta \delta}$ \\
\hline$k_{\mathrm{off}}^{i}$ & $k_{\mathrm{on}}^{i} e^{\beta \Delta \mu_{i}}$ \\
\hline$k_{\mathrm{hyd}, f}^{i}$ & $k_{\mathrm{hyd}, f}^{c} e^{-\beta \delta}$ \\
\hline$k_{\mathrm{hyd}, r}^{i}$ & $k_{\mathrm{hyd}, f}^{i} e^{-10}$ \\
\hline$k_{\mathrm{pol}}^{i}$ & $k_{\mathrm{pol}}^{c} e^{-\beta \delta}$ \\
\hline$k_{\mathrm{dep}}^{i}$ & $k_{\mathrm{pol}}^{i} e^{-8}$ \\
\hline$k_{\mathrm{PR}, f}^{i}$ & $\kappa \mathrm{PR} e^{-\beta \delta+\beta \Delta \mu_{i}} s^{-1}$ \\
\hline$k_{\mathrm{PR}, r}^{i}$ & $k_{\mathrm{PR}, f}^{i} e^{-8-\beta \Delta \mu_{i}} s^{-1}$ \\
\hline
\end{tabular}

